\title{
Hydrogeochemical study of spas groundwaters from southeast Brazil
}

\author{
Daniel Marcos Bonotto \\ Departamento de Petrologia e Metalogenia, Universidade Estadual Paulista (UNESP), Câmpus de Rio Claro, Av. 24-A No.1515, C.P. 178, CEP 13506-900 Rio Claro, São Paulo, Brazil
}

\section{A R T I C L E I N F O}

\section{Article history:}

Received 10 August 2015

Revised 17 March 2016

Accepted 17 July 2016

Available online 18 July 2016

Keywords:

Mineral waters

Spring waters

Hydrochemistry

Water classification

Southeast Brazil

\begin{abstract}
A B S T R A C T
This paper describes a hydrochemical study focusing spas groundwaters occurring at São Paulo and Minas Gerais states, Brazil, that are extensively used for drinking in public places, bottling and bathing purposes, among other. The water samples (75) for this study were taken from springs and pumped tubular wells drilled at different aquifer systems that are inserted in Paraná and Southeastern Shield hydrogeological provinces. The data acquisition for temperature, electrical conductivity (EC), pH, redox potential (Eh), dissolved gases $\left(\mathrm{O}_{2}, \mathrm{CO}_{2}\right.$ and $\mathrm{H}_{2} \mathrm{~S}$ ) and alkalinity was in situ performed for avoiding losses and modification due to transportation. The total dissolved solids (TDS) concentration was evaluated by gravimetry, the major cations and anions, iron and silica by colorimetry/atomic absorption spectrophotometry (AAS), and fluoride by potentiometry. The acquired database allowed establish the principal trends among the parameters analyzed after assuring its consistence from expected relationships found in hydrogeochemical surveys. The groundwaters are reducing (from $\mathrm{pH}$ and $\mathrm{Eh}$ data), there is a direct TDS-EC relationship, implying on a significant correlation between their ionic strength (IS) and EC. The major ions justifying such trends were sodium, (bi)carbonate, chloride, sulfate and phosphate that also correlated positively with the IS. The spas groundwaters were classified according to the guidelines of the Brazilian Code of Mineral Waters (BCMW) and EU directive for mineral waters. The major hydrochemical facies were also determined, as well the main sources influencing the groundwater composition and possible subsurface temperatures.
\end{abstract}

(C) 2016 Published by Elsevier B.V.

\section{Introduction}

In the last few decades, the consumption of natural drinking water, either spring or mineral (bottled or not), increased in several countries. Despite drinking water has been used mostly as the tap water accessible in every household, many people believe that the naturally occurring waters are healthy and/or can be utilized for health cures, thus, exhibiting better quality than the tap water. Additionally, economic reasons have also favored their use as bottled waters so that the commercialization of mineral waters has widely increased, inclusive in Brazil where circa 20 million consumers are involved (SEBRAE, 2012).

The thermal and mineral waters use in Brazil is not recent due to arrival of European immigrants, mainly from Portugal. The construction of thermal and non-thermal spas for therapeutic and leisure purposes reached a maximum number in the 1930s and 1950s, mainly at São Paulo (SP) and Minas Gerais (MG) states (Mourão, 1992). The Brazilian Code of Mineral Waters (BCMW) was established in this time, under French influence, by Register 7841 published on 8 August 1945 (DFPM, 1966). It classifies the mineral waters for spas and bottling uses, as well the potable waters for bottling, including several

E-mail address: danielmarcosbonotto@gmail.com. parameters like the radioactivity due to dissolved ${ }^{222} \mathrm{Rn}$ and ${ }^{220} \mathrm{Rn}$ (DFPM, 1966; Serra, 2009).

Some hydrogeochemical studies of Brazilian natural mineral waters took into account the BCMW guidelines. For instance, Bertolo et al. (2007) analyzed 303 labels of bottled mineral waters, grouping them according to the total dissolved solids (TDS) concentration. Oliveira et al. (2001) and Godoy et al. (2001) measured ${ }^{226} \mathrm{Ra},{ }^{228} \mathrm{Ra}$ and ${ }^{210} \mathrm{~Pb}$ in 17 and 28 brands of bottled mineral waters, respectively. Szikszay (1981) realized a detailed hydrogeochemical survey at Águas da Prata (SP) spa, whereas Oliveira et al. (1998) evaluated the ${ }^{222} \mathrm{Rn}$ e ${ }^{226} \mathrm{Ra}$ seasonal variation there.

EuroGeoSurveys (The Geochemistry Group of the European Geological Surveys) managed a common European sampling campaign of bottled mineral and spring waters (analysis of 884 samples for $>70$ chemical parameters in one laboratory) whose results were published in 2010 in the Special Issue "Mineral Waters of Europe" of Journal of Geochemical Exploration (v. 107, pp. 217-422). The hydrogeochemical study held within the framework of the project involved different approaches like analytical techniques, major constituents, trace elements, radionuclides, stable isotopes, mapping, waters classification, statistical treatment of hydrochemical data, human health, etc. Water sources were from Croatia (Peh et al., 2010), Estonia (Bityukova and Petersell, 2010), Germany (Birke et al., 2010a, 2010b), Greece (Demetriades, 2010; Dotsika et al., 2010), Hungary (Fugedi et al., 2010), Italy (Dinelli 
et al., 2010; Cicchella et al., 2010), Norway/Sweden/Finland/Iceland (Frengstad et al., 2010), Portugal (Lourenço et al., 2010), Serbia (Petrović et al., 2010), Slovakia (Dušan et al., 2010), and Slovenia (Brenčič and Vreča, 2010; Brenčič et al., 2010).

DNPM-National Department of Mineral Production manages in Brazil the production and commercialization of mineral waters. The amount of bottled mineral waters at SP in 2007 was higher than 1.5 billion liters (34\% of the total in the country) (CPRM, 2012). The actual accentuated consumption of spring and mineral waters for drinking purposes in Brazil requests a better understanding of their composition as the available information is sparse without considering the various lithologies involved and the adoption of standardized procedures for sampling and analyses. They are widely exploited from spas located at SP and MG due to historical reasons and the generation of a consistent database from the use of the same experimental steps allowed new hydrogeochemical insights and a comparison with other water sources occurring elsewhere.

\section{Study area}

The groundwater samples (75) were taken from springs and pumped tubular wells from 14 spas located in SP and MG (Fig. 1) at various geological contexts: Águas de São Pedro (3), Águas da Prata (7), Águas de Lindóia (7), Serra Negra (8), Lindóia (2), Termas de Ibirá (5), Águas de Santa Bárbara (1), Lambari (6), São Lourenço (8), Cambuquira (6), Caxambu (10), Poços de Caldas (6), Pocinhos do Rio Verde (4) and Araxá (2). The water sources were from different aquifer systems in the Paraná and Southeastern Shield hydrogeological provinces (Table 1).

The spas of Águas de São Pedro, Águas de Santa Bárbara and Termas de Ibirá are in the Paraná basin, a huge sedimentary area of southern Brazil, with extensions into Paraguay, Uruguay and Argentina. The stratigraphical record of this intracratonic basin shows a tendency towards continental depositional systems (Milani, 2004). In the Neo-Ordovician, marine strata deposited from the onset of sedimentation continued throughout the Devonian with oceanic influence up to the Carboniferous (Milani,

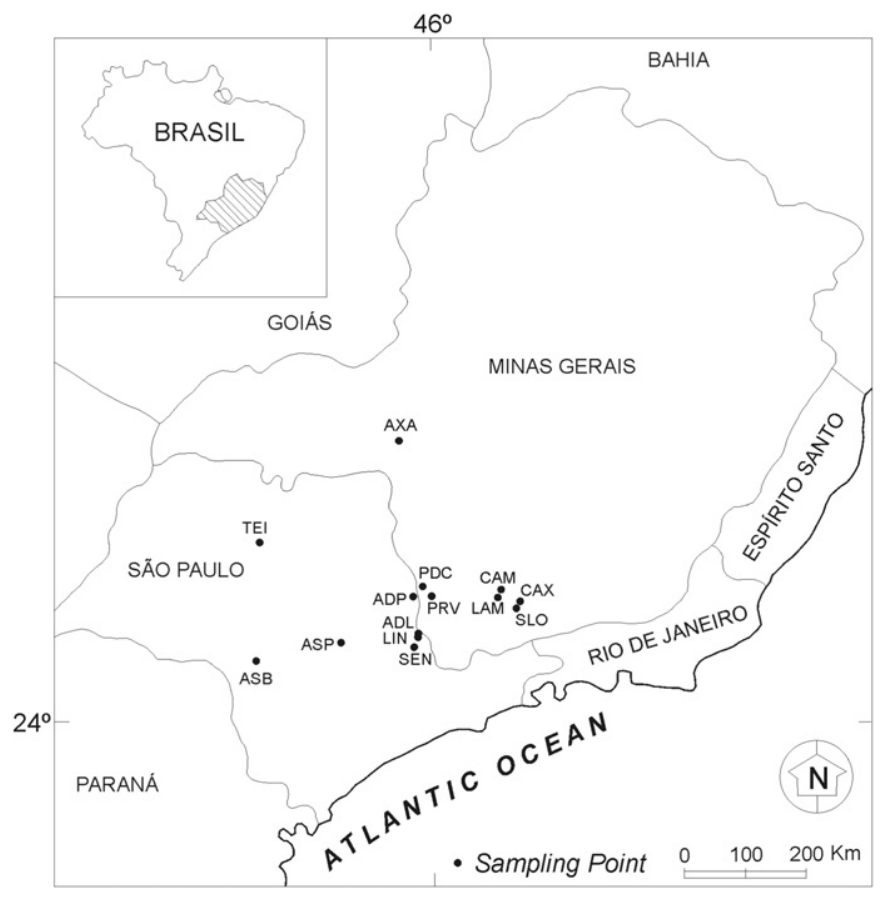

Fig. 1. Sketch map of the research region in Brazil and location of the groundwater sampling points in the following spas of São Paulo and Minas Gerais states: ASP = Águas de São Pedro, ADL = Águas de Lindóia, SEN = Serra Negra, LIN = Lindóia, TEI = Termas de Ibirá, ASB = Águas de Santa Bárbara, ADP = Águas da Prata, PDC = Poços de Caldas, PRV = Pocinhos do Rio Verde, $\mathrm{LAM}=$ Lambari, SLO = São Lourenço, CAM = Cambuquira, $\mathrm{CAX}=$ Caxambu, $\mathrm{AXA}=$ Araxá.
2004). From Permian times on, the basin took the form of a large inland sea (Milani, 2004). Eolian sandstone beds came to dominate the Mesozoic scenario and with the break-up of Gondwana, the basin basement was much affected by the intrusion of vast amounts of magma emplaced as dykes and sills between the sedimentary strata as well as flows on the surface (Milani, 2004). The evolutionary history of the Paraná basin closed in the Early-Cretaceous with the deposition of continental sediments over the Serra Geral lavas (Milani, 2004). Different magma eruption rates $\left(\sim 0.1 \mathrm{~km}^{3} \mathrm{yr}^{-1}\right.$ or $\left.\geq 2.0 \mathrm{~km}^{3} \mathrm{yr}^{-1}\right)$ and duration (11 Ma, from $140 \mathrm{Ma}$ to $129 \mathrm{Ma}$; $<1 \mathrm{Ma}$, began at $134.7 \mathrm{Ma}$ ) of the Paraná continental flood basalt (CFB) volcanism have been suggested from ${ }^{40} \mathrm{Ar} /{ }^{39} \mathrm{Ar}$ geochronology data (Renne et al., 1992; Turner et al., 1994; Thiede and Vasconcelos, 2010; etc.). The main rocks in the Paraná basin are sandstones, conglomerates, diamictites, tillites, siltstones, shales, rythmites, silex, mudstones, limestones, basalts and diabases (IPT, 1981). Deep tube wells have exploited groundwaters of the sampling points at Águas de Santa Bárbara spa (Serra Geral and Botucatu formations) and Águas de São Pedro spa (Tubarão Group) (Kimmelmann et al., 1987).

The spas of Águas de Lindóia, Serra Negra and Lindóia are in a region where various phases/cycles involving metamorphic, deformation and magmatic events have acted from the Archean to the Upper Proterozoic times. They have affected rocks of high metamorphic degree, generally of granulite and amphibolite facies (Ebert, 1955; Almeida and Hasui, 1984). The principal rocks occurring there are immature sediments (comprising sand, silt, clays and organic matter), milonites, quartzites, schists, gneisses, anfibolites, migmatites and syntectonic granites (Zanardo, 1987). The groundwater samples at Águas de Lindóia spa provided from fractures/fissures/faults occurring in migmatite (Lindália and Santa Isabel springs), quartzite (Comexim, Curie, Filomena and Beleza springs) and milonite/quartzite (São Roque spring) (del Rey, 1989).

The extensive Early-Cretaceous Paraná CFB province and a number of Early-Cretaceous to Eocene alkaline igneous provinces that surround the Paraná Basin like the Poços de Caldas alkaline massif (PCAM), Alto Paranaíba igneous province (APIP) and minor occurrences have been associated with the thermal and/or chemical influence of mantle-plumes (Tristan and Trindade) impacting on the base of the continental lithosphere (e.g. Gibson et al., 1995a, 1995b; Thompson et al., 1998).

The spas of Águas da Prata, Poços de Caldas and Pocinhos do Rio Verde are located in PCAM that is roughly circular (diameter $\sim 33 \mathrm{~km}$ ) and covers $\sim 800 \mathrm{~km}^{2}$. It is a suite of alkaline volcanic and plutonic rocks (mainly phonolites and nepheline syenites) whose evolutionary history starts with major early volcanism involving ankaratrites (biotite-bearing nephelinite), phonolite lavas, and volcano-clastics, followed by caldera subsidence and nepheline syenite intrusions forming minor ring dykes and circular structures and, finally, the intrusion of eudialite-bearing nepheline syenites (Ellert, 1959; Schorscher and Shea, 1992; Ulbrich et al., 2005). The following springs were sampled at Águas da Prata spa: Villela and Boi (discharges into sandstones); Vitória and Prata (discharges through fissures in diabase); Platina, Paiol and Padre (discharges through volcanic tuffs, phonolites and eudialite-bearing nepheline syenites) (Szikszay, 1981). Groundwaters at Poços de Caldas and Pocinhos do Rio Verde spas were from thermal/non-thermal springs discharging in crystalline fractured rocks (Cruz and Peixoto, 1989).

Araxá spa is located at APIP, including the renowned Araxá carbonatite circular intrusion (diameter $\sim 4.5 \mathrm{~km}$ ) (Traversa et al., 2001). The APIP is one of the world's most voluminous mafic potassic provinces composed of a relatively diverse suite of ultrapotassic-potassic, ultramafic-mafic, silica-undersaturated lavas and hypabyssal intrusions with very high concentrations of incompatible trace elements and strongly enriched in light rare earths relative to heavy rare earth elements (Gibson et al., 1995b; Gomes and Comin-Chiaramonti, 2005). A 
Table 1

Description of the water samples analyzed in this paper.

\begin{tabular}{|c|c|c|c|c|c|c|}
\hline City $\left(\right.$ State $\left.^{\mathrm{a}}\right)$ & $\begin{array}{l}\text { Spring (well) } \\
\text { name/sample code }\end{array}$ & $\begin{array}{l}\text { Hydrogeological } \\
\text { province }^{\mathrm{b}}\end{array}$ & $\begin{array}{l}\text { Dominant } \\
\text { flow }\end{array}$ & Major rock types & Geological context/age & Commercialization $^{c}$ \\
\hline Águas de São Pedro (SP) & $\begin{array}{l}\text { Almeida Salles/ALS } \\
\text { Gioconda/GIO } \\
\text { Juventude/JUV }\end{array}$ & Paraná & Porous & Sandstones & $\begin{array}{l}\text { Botucatu Fm. (Jurassic) } \\
\text { Pirambóia Fm. (Triassic) } \\
\text { Itararé Fm. (Permian) } \\
\text { Irati Fm. (Permian) }\end{array}$ & \\
\hline Águas da Prata (SP) & $\begin{array}{l}\text { Platina/PLA } \\
\text { Paiol/POL }\end{array}$ & Paraná & Fractures & $\begin{array}{l}\text { Diabases } \\
\text { Phonolites }\end{array}$ & $\begin{array}{l}\text { Botucatu Fm. (Jurassic) } \\
\text { Serra Geral Fm. } \\
\text { (Jurassic-Cretaceous) }\end{array}$ & Yes \\
\hline & $\begin{array}{l}\text { Vitória/VIT } \\
\text { Boi/BOI }\end{array}$ & & & $\begin{array}{l}\text { Alkaline rocks } \\
\text { Silicified sandstones }\end{array}$ & $\begin{array}{l}\text { Poços de Caldas intrusive complex } \\
\text { (Cretaceous) }\end{array}$ & \\
\hline & $\begin{array}{l}\text { Prata/PTA } \\
\text { Villela/VIL } \\
\text { Padre/PDE }\end{array}$ & & & & & Yes \\
\hline Águas de Lindóia (SP) & $\begin{array}{l}\text { Santa Isabel/SIL } \\
\text { Filomena/FIL } \\
\text { Beleza/BEL } \\
\text { São Roque/SRE } \\
\text { Comexim/COM } \\
\text { Lindália/LIN } \\
\text { Curie/CUR }\end{array}$ & Paraná & Fractures & $\begin{array}{l}\text { Granites } \\
\text { Gneisses } \\
\text { Migmatites } \\
\text { Schists } \\
\text { Quartzites } \\
\text { Limestones } \\
\text { Dolomites }\end{array}$ & Amparo Gp. (Lower Proterozoic) & Yes \\
\hline Serra Negra (SP) & $\begin{array}{l}\text { São Jorge/SJO } \\
\text { São Carlos/SCA } \\
\text { Italianos/ITA } \\
\text { Santa Luzia/SLU } \\
\text { Santo Agostinho/SAT } \\
\text { Brunhara/BRU } \\
\text { Laudo Natel/LAN } \\
\text { Sant'Anna/SAA }\end{array}$ & & & & & \\
\hline Lindóia (SP) & $\begin{array}{l}\text { São Benedito/SBE } \\
\text { Bioleve/BIO }\end{array}$ & & & & & $\begin{array}{l}\text { Yes } \\
\text { Yes }\end{array}$ \\
\hline Termas de Ibirá (SP) & $\begin{array}{l}\text { Jorrante/JOR } \\
\text { Ademar de Barros/ADB } \\
\text { Carlos Gomes/CGO } \\
\text { Saracura/SRC } \\
\text { Seixas/SEI }\end{array}$ & Paraná & $\begin{array}{l}\text { Porous } \\
\text { and } \\
\text { fractures }\end{array}$ & $\begin{array}{l}\text { Sandstones } \\
\text { Basalts }\end{array}$ & $\begin{array}{l}\text { Bauru Gp. (Cretaceous) } \\
\text { Serra Geral Fm. } \\
\text { (Jurassic-Cretaceous) }\end{array}$ & \\
\hline $\begin{array}{l}\text { Águas de Santa Bárbara } \\
\text { (SP) }\end{array}$ & $\begin{array}{l}\text { Balneário } \\
\text { Municipal/BMU }\end{array}$ & Paraná & $\begin{array}{l}\text { Porous } \\
\text { and } \\
\text { fractures }\end{array}$ & $\begin{array}{l}\text { Sandstones } \\
\text { Basalts }\end{array}$ & $\begin{array}{l}\text { Bauru Gp. (Cretaceous) } \\
\text { Serra Geral Fm. } \\
\text { (Jurassic-Cretaceous) }\end{array}$ & \\
\hline Lambari (MG) & $\begin{array}{l}\text { No. } 1 / \text { LA1 } \\
\text { No. 2/LA2 } \\
\text { No. 3/LA3 } \\
\text { No. 4/LA4 } \\
\text { No. 5/LA5 } \\
\text { No. 6/LA6 }\end{array}$ & $\begin{array}{l}\text { Southeastern } \\
\text { shield }\end{array}$ & $\begin{array}{l}\text { Porous } \\
\text { and } \\
\text { fractures }\end{array}$ & $\begin{array}{l}\text { Ortogneisses } \\
\text { Granulites } \\
\text { Migmatites } \\
\text { Metassedimentary seq. } \\
\text { Metavulcanossedimentary } \\
\text { seq. }\end{array}$ & $\begin{array}{l}\text { Paraíba do Sul Gp. (Proterozoic) } \\
\text { Barbacena Gp. (Proterozoic) } \\
\text { São João d'el Rei Gp. (Proterozoic) } \\
\text { Andrelândia Gp. (Proterozoic) } \\
\text { Magmatic plutonic series } \\
\text { (Brasiliano) }\end{array}$ & \\
\hline São Lourenço (MG) & $\begin{array}{l}\text { No. 7-Bis/SL7 } \\
\text { No. 5-Alcalina/SL5 } \\
\text { No. 6-Sulfurosa/SL6 } \\
\text { No. 3-Vichy/SL3 } \\
\text { No. 4-Ferruginosa/SL4 } \\
\text { No. 1-Oriente/SL1 } \\
\text { No. 10-Primavera/SL10 } \\
\text { No. 9-Carbogasosa/SL9 }\end{array}$ & & & & & \\
\hline Cambuquira (MG) & $\begin{array}{l}\text { Roxo Rodrigues/ROR } \\
\text { Regina Werneck/REW } \\
\text { Com. Augusto Ferreira/CAF } \\
\text { Fernandes Pinheiro/FEP } \\
\text { Marimbeiro/MAR } \\
\text { Souza Lima/SLI }\end{array}$ & & & & & \\
\hline Caxambu (MG) & $\begin{array}{l}\text { Geiser Floriano de } \\
\text { Lemos/GFL } \\
\text { Venâncio/VEN } \\
\text { Mayrink/MAY } \\
\text { Ernestina Guedes/EGU } \\
\text { Viotti/VIO } \\
\text { D. Pedro II/DPE } \\
\text { Beleza/BZA } \\
\text { Duque de Saxe/DXE } \\
\text { Da. Leopoldina/LEO } \\
\text { Da. Isabel/Conde d'Eu/ISA }\end{array}$ & & & & & \\
\hline
\end{tabular}


Table 1 (continued)

\begin{tabular}{|c|c|c|c|c|c|c|}
\hline City $\left(\right.$ State $\left.^{\mathrm{a}}\right)$ & $\begin{array}{l}\text { Spring (well) } \\
\text { name/sample code }\end{array}$ & $\begin{array}{l}\text { Hydrogeological } \\
\text { province }^{b}\end{array}$ & $\begin{array}{l}\text { Dominant } \\
\text { flow }\end{array}$ & Major rock types & Geological context/age & Commercialization $^{\mathrm{c}}$ \\
\hline Poços de Caldas (MG) & $\begin{array}{l}\text { Quisisana/QUI } \\
\text { XV de Novembro/NOV } \\
\text { Macacos/MAC } \\
\text { Sinhazinha/SIN } \\
\text { Frayha/FRA } \\
\text { Pedro Botelho/PEB }\end{array}$ & $\begin{array}{l}\text { Southeastern } \\
\text { shield }\end{array}$ & Fractures & $\begin{array}{l}\text { Alkaline rocks } \\
\text { Nepheline syenites } \\
\text { Phonolites } \\
\text { Pyroclastics } \\
\text { Volcanic tuffs }\end{array}$ & $\begin{array}{l}\text { Poços de Caldas intrusive complex } \\
\text { (Cretaceous) }\end{array}$ & \\
\hline $\begin{array}{l}\text { Pocinhos do Rio Verde } \\
\text { (MG) }\end{array}$ & $\begin{array}{l}\text { Rio Verde/RIV } \\
\text { Samaritana/SMA } \\
\text { São José/SJO } \\
\text { Amorosa/AMO }\end{array}$ & & & & & \\
\hline Araxá (MG) & $\begin{array}{l}\text { Dona Beja/DBJ } \\
\text { Andrade Júnior/AJU }\end{array}$ & $\begin{array}{l}\text { Southeastern } \\
\text { shield }\end{array}$ & $\begin{array}{l}\text { Porous } \\
\text { and } \\
\text { fractures }\end{array}$ & $\begin{array}{l}\text { Quartzites and schists } \\
\text { Alkaline-carbonatitic rocks }\end{array}$ & $\begin{array}{l}\text { Cretaceous } \\
\text { PreCambrian }\end{array}$ & \\
\hline
\end{tabular}

a $\mathrm{SP}=$ São Paulo State, $\mathrm{MG}=$ Minas Gerais State.

b According to Mente (2008).

c Water commercialized by private company in addition to consumption in taps accessible to population in public areas.

complex network of carbonatite as concentric and radial dykes quite variable in dimension and also small veins ranging from few millimeters to several centimeters in thickness are present in the region of Araxá spa, intruding either alkaline or country rocks. Additional lithologies include mica-rich rocks, phoscorites and lamprophyres (Traversa et al., 2001). Two springs were sampled at Araxá spa: 1) Dona Beja, associated to an aquifer system classified as granular, free and semi-confined, mainly in the intrusive body domain (Beato et al., 2000); 2) Andrade Júnior, related to a deep fractured aquifer, unconfined to semi-confined, mainly occurring in rocks surrounding the carbonatite complex (Beato et al., 2000).

Minor alkaline occurrences representing multi-stage intrusions emplaced into Late-Proterozoic metamorphic rocks were reported by CPRM $(1999,2008)$ during mapping of south MG where are located Lambari, São Lourenço, Cambuquira and Caxambu spas. The main rocks in the region are biotite gneisses, migmatized granitoids, protomilonites, milonite gneisses, metabasites intercalations secondarily cut by pegmatoids veins, schists, weathered quartzites and alluvial deposits. The gneissic rocks in Caxambu hill are cut by mafic dykes and alkaline breccias, constituting important recharge areas of the fractured aquifers (CPRM, 1999). In São Lourenço, Lambari and Caxambu spas, the rainwater infiltration in weathered horizons of gneissic rocks is followed by percolation through milonitized zones and fractures partially filled by pegmatoids dykes/alkaline breccias (CPRM, 1999). The periodical eruption of a non-geothermal geyser ( $\sim$-m high) occurs at Caxambu spa due to the pressure build-up from dissolved $\mathrm{CO}_{2}$ in water.

\section{Experimental}

One groundwater sample from each point was collected during the dry season (June-September) for avoiding contribution of recent recharged rainwater. This limited number of samples was due to the size of the study area and costs involved for field trips and analyses. With exception of geyser "Floriano de Lemos" (code GFL), all water sources have been used for drinking purposes, whilst some have been commercialized by private companies under different brands (Table 1).

The data acquisition for temperature, electrical conductivity (EC), $\mathrm{pH}$, redox potential Eh, dissolved gases $\left(\mathrm{O}_{2}, \mathrm{CO}_{2}\right.$ and $\left.\mathrm{H}_{2} \mathrm{~S}\right)$ and alkalinity happened in situ for avoiding losses/modification due to transportation. The groundwater samples $(\sim 3 \mathrm{~L})$ were collected from taps/pipes installed in each spring/well, stored in polyethylene bottles and transported to LABIDRO-Isotopes and Hydrochemistry Laboratory, Rio Claro city, for chemical analysis. Each sample was divided into different aliquots and unfiltered + unpreserved or filtered through $0.45 \mu \mathrm{m}$
Millipore membrane + preserved with different acids, depending on the requirements of the analyses.

Digital meters were used for recording the following parameters: temperature - through a thermocouple sensor; $\mathrm{pH}$ - through a combination glass electrode and buffer solutions for calibration; Eh - through a combination Pt electrode- $\mathrm{Ag} / \mathrm{AgCl}$ reference element and a Zobell I (Zobell, 1946) standard solution; EC - through a $1 \mathrm{~cm}^{2}$ area Pt electrode calibrated with $\mathrm{KCl}$ standards; dissolved oxygen (DO) concentration through a $\mathrm{O}_{2}$ sensible electrode, i.e. a metallic wire covered by a thin Au layer; fluoride - through an ion-selective electrode and standards with variable $\mathrm{F}^{-}$concentrations. The Eh readings were done adopting the procedure described by Bonotto (2006), whereas all DO data were properly corrected due to altitude differences of the sampling points (Hach, 1992).

The total alkalinity (ALK) was determined by titration to an endpoint evidenced by the color change of a standard indicator solution of the bicarbonate, carbonate and hydroxide concentrations (APHA, 1989; Hach, 1992). Titration with phenolphthalein indicator $+0.0227 \mathrm{~N} \mathrm{NaOH}$ solution was adopted to evaluate the dissolved $\mathrm{CO}_{2}$ (in $\mathrm{mg} / \mathrm{L}$ ) from the titrant volume used (in $\mathrm{mL}$ ) (Hach, 1992). The dissolved $\mathrm{CO}_{2}$ corresponded to $0 \mathrm{mg} / \mathrm{L}$ when the color changed after the indicator addition, without the need of adding titrant (Hach, 1992).

The dissolved sulfide gas (in $\mathrm{mg} / \mathrm{L}$ sulfide, $\mathrm{S}^{2-}$ ) was determined by colorimetry (wavelength $665 \mathrm{~nm}$ ) using a program stored in the Hach DR/2000 spectrophotometer (Hach, 1992). The methylene blue method was adopted in which the $\mathrm{H}_{2} \mathrm{~S}$ and acid-soluble metal sulfides reacted with $N, N$-dimethyl- $p$-phenylenediamine oxalate to form methylene blue (Hach, 1992). The intensity of the blue color was proportional to the $S^{2-}$ concentration, whilst dilutions were used to determine high levels in some samples.

The dry residue (DR) ( TDS, total dissolved solids) content was evaluated by evaporation and weighing (APHA, 1989). Atomic absorption spectrophotometry (AAS) was used to measure the dissolved $\mathrm{Na}^{+}$concentration, whereas colorimetry for characterizing the dissolved $\mathrm{K}^{+}$, $\mathrm{Ca}^{2+}, \mathrm{Mg}^{2+}, \mathrm{Cl}^{-}, \mathrm{NO}_{3}^{-}, \mathrm{SO}_{4}^{2-}, \mathrm{PO}_{4}^{3-}, \mathrm{Fe}, \mathrm{Fe}^{2+}$ and $\mathrm{SiO}_{2}$ concentrations. Various reagents added to the samples produced colored complexes read by a program stored in Hach DR/2000 spectrophotometer that had been previously calibrated (Hach, 1992). Table 2 summarizes the methods adopted and respective detection limits.

\section{Results}

Tables 3 and 4 report all data obtained. Aquachem 4.0 software (Waterloo Hydrogeologic, 2003) evaluated the ionic strength (IS) of the waters (Table 3 ) and performed the statistical data treatment (Table 5). 
Table 2

Analytical methods and detection limits.

\begin{tabular}{|c|c|c|c|c|c|c|c|}
\hline Parameter & Unit & Analytical method & Detection limit & Parameter & Unit & Analytical method & Detection limit \\
\hline Temp. & ${ }^{\circ} \mathrm{C}$ & Potentiometric & - & $\mathrm{Na}$ & $\mu \mathrm{g} / \mathrm{L}$ & AAS & 0.3 \\
\hline $\mathrm{pH}$ & - & Potentiometric & - & K & $\mathrm{mg} / \mathrm{L}$ & Colorimetric & 0.13 \\
\hline Eh & $\mathrm{mV}$ & Potentiometric & - & $\mathrm{Ca}$ & $\mu \mathrm{g} / \mathrm{L}$ & Colorimetric & 20 \\
\hline EC & $\mu \mathrm{S} / \mathrm{cm}$ & Conductometric & - & $\mathrm{Mg}$ & $\mu \mathrm{g} / \mathrm{L}$ & Colorimetric & 6 \\
\hline DO & $\mathrm{mg} / \mathrm{L}$ & Potentiometric & 0.1 & $\mathrm{SiO}_{2}$ & $\mathrm{mg} / \mathrm{L}$ & Colorimetric & 0.45 \\
\hline $\mathrm{CO}_{2}$ & $\mathrm{mg} / \mathrm{L}$ & Titration & - & $\mathrm{Cl}^{-}$ & $\mathrm{mg} / \mathrm{L}$ & Colorimetric & 0.3 \\
\hline $\mathrm{H}_{2} \mathrm{~S}$ & $\mu \mathrm{g} / \mathrm{L}$ & Colorimetric & 1 & $\mathrm{~F}^{-}$ & $\mu \mathrm{g} / \mathrm{L}$ & Potentiometric & 20 \\
\hline TDS & $\mathrm{mg} / \mathrm{L}$ & Gravimetric & 1 & $\mathrm{NO}_{3}^{-}$ & $\mathrm{mg} / \mathrm{L}$ & Colorimetric & 0.8 \\
\hline ALK & $\mathrm{mg} / \mathrm{L}$ & Titration & 1 & $\mathrm{SO}_{4}^{2-}$ & $\mathrm{mg} / \mathrm{L}$ & Colorimetric & 0.5 \\
\hline $\mathrm{Fe}_{\text {tot }}$ & $\mu \mathrm{g} / \mathrm{L}$ & Colorimetric & 6 & $\mathrm{PO}_{4}^{3-}$ & $\mu \mathrm{g} / \mathrm{L}$ & Colorimetric & 20 \\
\hline $\mathrm{Fe}^{2+}$ & $\mu \mathrm{g} / \mathrm{L}$ & Colorimetric & 6 & & & & \\
\hline
\end{tabular}

$\mathrm{EC}=$ electrical conductivity; $\mathrm{DO}=$ dissolved oxygen; TDS = total dissolved solids; ALK = total alkalinity; AAS = atomic absorption spectroscopy.

Table 3

Physicochemical data of the waters analyzed in this study.

\begin{tabular}{|c|c|c|c|c|c|c|c|c|c|c|c|c|c|}
\hline $\begin{array}{l}\text { Sample } \\
\text { code }\end{array}$ & $\begin{array}{l}\text { Temp. }\left(^{\circ}\right. \\
\text { C) }\end{array}$ & $\mathrm{pH}$ & $\begin{array}{l}\text { Eh } \\
(\mathrm{mV})\end{array}$ & $\begin{array}{l}\mathrm{EC} \\
(\mu \mathrm{S} / \mathrm{cm})\end{array}$ & $\begin{array}{l}\mathrm{DO} \\
(\mathrm{mg} / \mathrm{L})\end{array}$ & $\begin{array}{l}\mathrm{CO}_{2} \\
(\mathrm{mg} / \mathrm{L})\end{array}$ & $\begin{array}{l}\mathrm{H}_{2} \mathrm{~S} \\
(\mu \mathrm{g} / \mathrm{L})\end{array}$ & $\begin{array}{l}\text { ALK } \\
(\mathrm{mg} / \mathrm{L})\end{array}$ & $\begin{array}{l}\mathrm{SiO}_{2} \\
(\mathrm{mg} / \mathrm{L})\end{array}$ & $\begin{array}{l}\mathrm{Fe}_{\text {tot }} \\
(\mathrm{mg} / \mathrm{L})\end{array}$ & $\begin{array}{l}\mathrm{Fe}^{2+} \\
(\mathrm{mg} / \mathrm{L})\end{array}$ & $\begin{array}{l}\text { TDS } \\
(\mathrm{mg} / \mathrm{L})\end{array}$ & $\begin{array}{l}\text { IS } \\
\left(\times 10^{-4}\right)\end{array}$ \\
\hline ALS & 27.1 & 7.40 & 16 & 710 & 3.13 & 120 & 3 & 261 & 39.7 & 0.03 & b.d. & 1880 & 30.8 \\
\hline GIO & 27.4 & 8.45 & -59 & 3790 & 5.08 & 100 & 6 & 204 & 12.8 & 0.01 & b.d. & 1700 & 167.7 \\
\hline JUV & 26.7 & 8.59 & -59 & 4730 & 2.81 & 88 & 3064 & 338 & 24.5 & 0.66 & b.d. & 1960 & 189.5 \\
\hline PLA & 25.4 & 8.21 & -5 & 1530 & 1.33 & 400 & 5 & 472 & 33.1 & 0.01 & b.d. & 936 & 101.0 \\
\hline POL & 25.8 & 8.23 & -37 & 3630 & 3.06 & 800 & 12 & 1390 & 29.0 & 0.02 & b.d. & 2625 & 260.8 \\
\hline VIT & 24.5 & 9.23 & -150 & 3370 & 1.33 & 1032 & 2 & 1388 & 32.8 & 0.06 & b.d. & 1042 & 311.3 \\
\hline BOI & 24.2 & 7.57 & -154 & 40 & 3.77 & 128 & 4 & 17 & 18.6 & 0.22 & 0.01 & 54 & 9.8 \\
\hline PTA & 25.1 & 7.17 & -25 & 280 & 7.14 & 180 & 4 & 73 & 19.7 & 0.01 & b.d. & 2215 & 11.4 \\
\hline VIL & 24.3 & 7.98 & -159 & 30 & 5.71 & 140 & 3 & 2 & 8.9 & 0.05 & b.d. & 43 & 4.9 \\
\hline PDE & 23.7 & 6.74 & -52 & 220 & 5.10 & 256 & 4 & 99 & 19.3 & b.d. & b.d. & 240 & 13.5 \\
\hline SIL & 22.5 & 6.94 & -27 & 160 & 5.35 & 96 & 1 & 36 & 17.0 & b.d. & b.d. & 59 & 6.2 \\
\hline FIL & 26.9 & 7.86 & -33 & 220 & 4.65 & 108 & 2 & 65 & 14.1 & 0.05 & b.d. & 38 & 8.8 \\
\hline BEL & 26.4 & 7.89 & -17 & 210 & 3.84 & 88 & 2 & 69 & 17.3 & 0.11 & b.d. & 89 & 8.3 \\
\hline SRE & 27.3 & 7.52 & 6 & 230 & 4.44 & 164 & 2 & 58 & 15.7 & 0.02 & b.d. & 74 & 7.3 \\
\hline $\mathrm{COM}$ & 23.6 & 5.61 & 97 & 20 & 6.06 & 100 & 1 & 11 & 12.3 & 0.15 & b.d. & 11 & 3.1 \\
\hline LIN & 24.6 & 6.98 & -3 & 360 & 5.66 & 136 & 4 & 76 & 37.5 & 0.02 & 0.02 & 86 & 17.4 \\
\hline CUR & 26.2 & 6.86 & -38 & 180 & 4.65 & 128 & 5 & 60 & 17.6 & 0.01 & b.d. & 154 & 8.8 \\
\hline SJO & 22.0 & 5.92 & -54 & 140 & 6.16 & 132 & 2 & 16 & 18.5 & b.d. & b.d. & 172 & 7.6 \\
\hline SCA & 21.7 & 5.85 & 7 & 220 & 5.66 & 236 & b.d. & 71 & 34.3 & 0.01 & b.d. & 233 & 11.2 \\
\hline ITA & 20.4 & 6.18 & -38 & 210 & 5.66 & 212 & 8 & 62 & 34.1 & b.d. & b.d. & 193 & 10.4 \\
\hline SLU & 21.5 & 5.94 & -25 & 130 & 2.83 & 188 & 1 & 35 & 25.3 & 0.02 & b.d. & 225 & 8.0 \\
\hline SAT & 21.8 & 5.87 & -30 & 170 & 3.23 & 236 & 2 & 43 & 28.8 & b.d. & b.d. & 167 & 9.3 \\
\hline BRU & 22.1 & 5.94 & -28 & 180 & 4.85 & 160 & 1 & 27 & 32.5 & b.d. & b.d. & 144 & 9.0 \\
\hline LAN & 23.1 & 5.62 & -1 & 130 & 3.64 & 212 & 3 & 21 & 20.1 & b.d. & b.d. & 106 & 8.3 \\
\hline SAA & 21.9 & 6.07 & -55 & 150 & 4.65 & 200 & 1 & 52 & 35.0 & b.d. & b.d. & 185 & 9.6 \\
\hline SBE & 24.9 & 6.47 & -50 & 290 & 9.05 & 180 & 3 & 83 & 40.6 & 0.12 & b.d. & 286 & 13.6 \\
\hline $\mathrm{BIO}$ & 23.6 & 5.96 & -74 & 90 & 6.45 & 132 & 3 & 24 & 20.3 & 0.14 & b.d. & 134 & 6.5 \\
\hline JOR & 26.8 & 9.03 & -144 & 660 & 2.05 & 0 & 13 & 99 & 34.3 & 0.01 & b.d. & 410 & 52.8 \\
\hline $\mathrm{ADB}$ & 28.4 & 8.96 & -145 & 670 & 2.59 & 0 & 3 & 130 & 32.4 & 0.01 & b.d. & 420 & 51.9 \\
\hline CGO & 28.4 & 9.09 & -147 & 680 & 1.30 & 0 & 5 & 98 & 34.6 & 0.05 & b.d. & 380 & 46.1 \\
\hline SRC & 28.7 & 9.17 & -150 & 700 & 0.97 & 0 & 35 & 90 & 33.4 & b.d. & b.d. & 460 & 41.9 \\
\hline SEI & 26.4 & 8.97 & -138 & 640 & 2.38 & 0 & 8 & 105 & 37.3 & b.d. & b.d. & 455 & 44.7 \\
\hline BMU & 24.6 & 7.58 & 112 & 160 & 5.99 & 252 & 2 & 73 & 39.2 & b.d. & b.d. & 200 & 21.6 \\
\hline LA1 & 23.0 & 4.55 & -90 & 190 & 5.00 & 1840 & 1 & 25 & 11.5 & 0.01 & b.d. & 121 & 8.3 \\
\hline LA2 & 23.0 & 4.53 & -91 & 190 & 4.69 & 1600 & 2 & 19 & 12.7 & 0.04 & 0.01 & 212 & 7.9 \\
\hline LA3 & 23.1 & 4.90 & -87 & 220 & 4.49 & 1480 & 1 & 28 & 11.7 & 0.02 & 0.01 & 198 & 8.9 \\
\hline LA4 & 24.0 & 4.16 & 32 & 190 & 4.69 & 600 & b.d. & 34 & 9.6 & 0.02 & 0.01 & 256 & 11.2 \\
\hline LA5 & 25.4 & 4.71 & -50 & 170 & 6.73 & 1840 & b.d. & 22 & 17.0 & 1.14 & 0.01 & 446 & 7.4 \\
\hline LA6 & 24.0 & 4.81 & -67 & 160 & 5.51 & 1480 & b.d. & 21 & 14.5 & 0.12 & b.d. & 139 & 6.5 \\
\hline SL7 & 24.7 & 6.17 & -67 & 620 & 1.53 & 800 & 18 & 158 & 34.8 & 1.55 & 0.02 & 198 & 24.7 \\
\hline SL5 & 23.6 & 6.38 & -56 & 1030 & 1.73 & 1560 & b.d. & 262 & 23.2 & 0.19 & b.d. & 185 & 37.8 \\
\hline SL6 & 25.2 & 6.18 & -39 & 1180 & 1.22 & 1720 & b.d. & 292 & 41.0 & 5.18 & 0.03 & 323 & 42.6 \\
\hline SL3 & 26.7 & 6.59 & -64 & 1050 & 3.67 & 1320 & b.d. & 303 & 38.7 & 1.10 & b.d. & 214 & 39.9 \\
\hline SL4 & 26.6 & 6.06 & -65 & 660 & 1.94 & 920 & b.d. & 93 & 21.0 & 1.15 & b.d. & 305 & 20.8 \\
\hline SL1 & 25.6 & 5.97 & -44 & 670 & 1.33 & 1420 & b.d. & 163 & 17.9 & 0.03 & 0.01 & 296 & 27.6 \\
\hline SL10 & 25.2 & 6.59 & -58 & 1710 & 3.06 & 1200 & b.d. & 495 & 34.4 & 0.09 & b.d. & 176 & 66.9 \\
\hline SL9 & 23.9 & 5.94 & -57 & 640 & 1.02 & 1480 & b.d. & 162 & 21.3 & 3.66 & 0.04 & 295 & 28.3 \\
\hline ROR & 24.0 & 4.90 & -37 & 90 & 3.74 & 800 & 1 & 2 & 18.6 & 0.05 & 0.01 & 126 & 4.4 \\
\hline REW & 24.0 & 4.64 & -23 & 90 & 3.03 & 1380 & 3 & 4 & 15.4 & 0.04 & 0.02 & 73 & 4.4 \\
\hline CAF & 26.3 & 4.60 & -38 & 100 & 4.34 & 1000 & 1 & 1 & 11.7 & 0.05 & 0.03 & 40 & 4.0 \\
\hline FEP & 25.0 & 5.50 & -35 & 240 & 2.63 & 1760 & 40 & 50 & 61.9 & 2.07 & 0.33 & 257 & 12.2 \\
\hline MAR & 25.5 & 5.76 & -42 & 570 & 3.33 & 1200 & 4 & 130 & 60.9 & 2.33 & 0.02 & 249 & 19.9 \\
\hline SLI & 23.2 & 5.30 & -24 & 170 & 3.13 & 1080 & 13 & 31 & 16.4 & 4.26 & 2.54 & 82 & 8.2 \\
\hline GFL & 23.8 & 7.00 & -39 & 2440 & 4.69 & 1100 & 14 & 919 & 44.2 & 1.11 & 0.99 & 1241 & 106.2 \\
\hline VEN & 25.0 & 6.43 & -51 & 2420 & 2.86 & 1680 & 17 & 784 & 38.9 & 0.27 & 0.23 & 757 & 92.1 \\
\hline
\end{tabular}


Table 3 (continued)

\begin{tabular}{|c|c|c|c|c|c|c|c|c|c|c|c|c|c|}
\hline $\begin{array}{l}\text { Sample } \\
\text { code }\end{array}$ & $\begin{array}{l}\text { Temp. }\left(^{\circ}\right. \\
\text { C) }\end{array}$ & $\mathrm{pH}$ & $\begin{array}{l}\text { Eh } \\
(\mathrm{mV})\end{array}$ & $\begin{array}{l}\mathrm{EC} \\
(\mu \mathrm{S} / \mathrm{cm})\end{array}$ & $\begin{array}{l}\text { DO } \\
(\mathrm{mg} / \mathrm{L})\end{array}$ & $\begin{array}{l}\mathrm{CO}_{2} \\
(\mathrm{mg} / \mathrm{L})\end{array}$ & $\begin{array}{l}\mathrm{H}_{2} \mathrm{~S} \\
(\mu \mathrm{g} / \mathrm{L})\end{array}$ & $\begin{array}{l}\text { ALK } \\
(\mathrm{mg} / \mathrm{L})\end{array}$ & $\begin{array}{l}\mathrm{SiO}_{2} \\
(\mathrm{mg} / \mathrm{L})\end{array}$ & $\begin{array}{l}\mathrm{Fe}_{\text {tot }} \\
(\mathrm{mg} / \mathrm{L})\end{array}$ & $\begin{array}{l}\mathrm{Fe}^{2+} \\
(\mathrm{mg} / \mathrm{L})\end{array}$ & $\begin{array}{l}\text { TDS } \\
(\mathrm{mg} / \mathrm{L})\end{array}$ & $\begin{array}{l}\text { IS } \\
\left(\times 10^{-4}\right)\end{array}$ \\
\hline MAY & 25.5 & 5.29 & -26 & 260 & 4.18 & 800 & 4 & 75 & 20.7 & 0.09 & 0.02 & 221 & 12.8 \\
\hline EGU & 25.8 & 6.25 & -68 & 2470 & 1.73 & 1600 & b.d. & 755 & 55.0 & 1.66 & 0.06 & 516 & 91.8 \\
\hline VIO & 25.3 & 5.40 & -59 & 400 & 1.84 & 800 & 15 & 130 & 23.4 & 0.03 & b.d. & 179 & 18.2 \\
\hline DPE & 24.7 & 5.59 & -66 & 620 & 1.94 & 880 & 9 & 153 & 21.7 & 0.03 & b.d. & 170 & 22.0 \\
\hline BZA & 23.9 & 6.41 & -65 & 3010 & 1.84 & 1440 & 1 & 680 & 60.2 & 3.18 & 2.94 & 757 & 94.3 \\
\hline DXE & 25.4 & 6.23 & -27 & 2040 & 1.84 & 720 & 7 & 643 & 42.3 & 0.11 & 0.09 & 820 & 76.0 \\
\hline LEO & 24.5 & 5.97 & -52 & 980 & 1.02 & 680 & 47 & 253 & 43.5 & 0.05 & 0.01 & 315 & 32.7 \\
\hline ISA & 24.2 & 5.99 & -74 & 2020 & 1.53 & 920 & 10 & 560 & 63.5 & 3.86 & 0.10 & 700 & 68.0 \\
\hline QUI & 22.4 & 6.13 & -41 & 170 & 2.25 & 104 & 1 & 10 & 9.9 & 0.04 & b.d. & 38 & 20.9 \\
\hline NOV & 25.7 & 9.38 & -53 & 920 & 1.37 & 0 & 259 & 196 & 28.4 & 0.04 & b.d. & 398 & 74.3 \\
\hline MAC & 32.1 & 9.60 & -51 & 1450 & 0.78 & 0 & 1184 & 233 & 31.0 & 0.01 & b.d. & 579 & 96.4 \\
\hline SIN & 25.7 & 9.54 & -90 & 1420 & 2.94 & 140 & 3 & 261 & 32.2 & 0.02 & b.d. & 574 & 114.1 \\
\hline FRA & 23.2 & 6.91 & -27 & 50 & 3.82 & 52 & b.d. & 6 & 6.7 & 0.14 & b.d. & 70 & 5.8 \\
\hline PEB & 35.7 & 9.58 & -70 & 1400 & 1.18 & 0 & 383 & 252 & 28.5 & 0.06 & b.d. & 600 & 117.2 \\
\hline RIV & 23.3 & 9.10 & -133 & 1290 & 1.30 & 180 & 756 & 270 & 30.5 & 0.02 & b.d. & 567 & 106.9 \\
\hline SMA & 23.9 & 9.05 & -133 & 1300 & 1.40 & 80 & 522 & 291 & 28.3 & b.d. & b.d. & 773 & 107.9 \\
\hline SJO & 22.0 & 8.98 & -133 & 1290 & 1.30 & 60 & 528 & 318 & 29.3 & b.d. & b.d. & 424 & 109.5 \\
\hline AMO & 21.7 & 5.56 & -18 & 40 & 5.80 & 112 & 4 & 5 & 11.0 & b.d. & b.d. & 48 & 3.2 \\
\hline DBJ & 22.1 & 7.50 & -146 & 330 & 5.00 & 252 & 1 & 112 & 23.4 & 0.06 & b.d. & 70 & 14.0 \\
\hline AJU & 29.0 & 9.57 & -141 & 6390 & 1.60 & 0 & 1980 & 2212 & 20.3 & 0.04 & b.d. & 2898 & 793.6 \\
\hline
\end{tabular}

EC = electrical conductivity; DO = dissolved oxygen; ALK = total alkalinity; TDS = total dissolved solids; IS = ionic strength; b.d. = below detection limit.

The criterion generally adopted to check the reliability and completeness of the hydrogeochemical data is that the deviation of the neutral ion balance should be lower than 5\% (Schoeller, 1962; Custodio and Llamas, 1976; Appelo and Postma, 2004). Aquachem 4.0 software (Waterloo Hydrogeologic, 2003) allowed find such values in only 4 water sources, possibly due to errors in the hydrogen carbonate determination, effects of waters containing very low to low mineral concentration and accentuated $\mathrm{SiO}_{2}$ contribution in the hydrochemistry. Silica is high in all waters analyzed as shown in the Schoeller (1962) diagrams plotted in Figs. 2 and 3 according to the four TDS classes defined by the EU mineral waters directive (van der $\mathrm{Aa}, 2003$ ). Despite $\mathrm{SiO}_{2}$ is not taken into account on the "neutrality condition" evaluation (Schoeller, 1962; Custodio and Llamas, 1976; Appelo and Postma, 2004), the quartz solubility increases rapidly at $\mathrm{pH}>9$, causing the silicic acid $\left(\mathrm{H}_{4} \mathrm{SiO}_{4}\right)$ deprotonation and forming the anion $\mathrm{H}_{3} \mathrm{SiO}_{4}^{-}$. Ritter (2012) identified this process when used the PHREEQC software for calculating the concentration of dominant solutes in spring waters of Poços de Caldas (MG) spa.

High-fluoride groundwaters are a recognized feature of a number of aquifers across the world (Smedley et al., 2002; Reddy et al., 2010; Edmunds and Smedley, 2013; etc.). The $\mathrm{F}^{-} / \mathrm{Cl}^{-}$molar ratios $>1$ in several water sources from PCAM indicate that $\mathrm{F}^{-}$is dominant relative to $\mathrm{Cl}^{-}$, whilst its importance is highlighted in the Piper (1944) diagram shown in Fig. 4. It also shows that bi (carbonate) and sodium ions dominate the water sources as confirmed by the following hydrochemical facies from the Aquachem 4.0 software (Waterloo Hydrogeologic, 2003): mixed (cations) $-\mathrm{HCO}_{3}^{-}, \mathrm{Na}^{+}$-mixed (anions), $\mathrm{Na}^{+}-\mathrm{HCO}_{3}^{-}, \mathrm{Ca}^{2+}-\mathrm{HCO}_{3}^{-}, \mathrm{Na}^{+}-$ $\mathrm{CO}_{3}^{2-}$ and $\mathrm{Na}^{+}-\mathrm{HCO}_{3}^{-}-\mathrm{CO}_{3}^{2-}$. Additionally, other water types also occur like mixed (cations and anions), mixed (cations) $-\mathrm{Cl}^{-}$and $\mathrm{K}^{+}-$ mixed (anions).

\subsection{Groundwater classification}

The BCMW establishes different waters classification based on temperature and chemical aspects (DFPM, 1966). According to temperature, they are cold $\left(25^{\circ} \mathrm{C}, 41\right.$ water sources - 54.7\%), hypothermal (25-33 ${ }^{\circ} \mathrm{C}$, 33 water sources $\left.-44 \%\right)$, and mesothermal $\left(33-36{ }^{\circ} \mathrm{C}, 1\right.$ water source $-1.3 \%$ ). Other classes defined by the BCMW are (DFPM, 1966): radiferous, radioactive, thoriferous, carbogaseous, bicarbonatealkaline, earth-alkaline, sulfated, sulfured, nitrated, chlorinated and ferruginous. Among these criteria, the BCMW guidelines for sulfur (dissolved sulfur $>1 \mathrm{mg} / \mathrm{L}$ ) and iron (dissolved iron $>5 \mathrm{mg} / \mathrm{L}$ ) suggest that the springs JUV (Águas de São Pedro-SP), MAC (Poços de Caldas-MG) and AJU (Araxá-MG) are sulfured, whereas the spring SL6 (São Lourenço-MG) is ferruginous.

The BCMW differs of the EU mineral water directive adopted by various European countries during the evaluation of the EuroGeoSurveys database (van der Aa, 2003). It will be also used here and, from the TDS classes, the waters analyzed in this research exhibit very low mineral concentration (TDS $<50 \mathrm{mg} / \mathrm{L}-6$ samples, $8 \%$ ), low mineral concentration (TDS $50-500 \mathrm{mg} / \mathrm{L}$ - 50 samples, 66.7\%), intermediate mineral concentration (TDS 500-1500 mg/L - 13 samples, 17.3\%), and high mineral concentration (TDS $>1500 \mathrm{mg} / \mathrm{L}-6$ samples, $8 \%$ ).

Additionally, the EU directive (van der Aa, 2003) allows classify the water sources as: containing bicarbonate $\left(\mathrm{HCO}_{3}^{-}>600 \mathrm{mg} / \mathrm{L}-9.3 \%\right.$, sample codes POL, VIT, GFL, VEN, EGU, BZA and DXE), containing sulfate $\left(\mathrm{SO}_{4}^{2-}>200 \mathrm{mg} / \mathrm{L}-1.3 \%\right.$, sample code GIO), containing iron $\left(\mathrm{Fe}^{2+}>1 \mathrm{mg} / \mathrm{L}-4 \%\right.$, sample codes SLI, GFL an BZA), containing sodium $\left(\mathrm{Na}^{+}>200 \mathrm{mg} / \mathrm{L}-8 \%\right.$, sample codes GIO, JUV, PLA, POL, VIT and AJU), containing fluoride $\left(\mathrm{F}^{-}>1 \mathrm{mg} / \mathrm{L}-32 \%, 24\right.$ water sources), acid $\left(\mathrm{CO}_{2}>250 \mathrm{mg} / \mathrm{L}-48 \%, 36\right.$ water sources $)$, and suitable for low sodium diets ( $\mathrm{Na}^{+}<20 \mathrm{mg} / \mathrm{L}-54.7 \%, 41$ water sources). Therefore, most of the spas groundwaters are acid and suitable for low sodium diets, various contain fluoride and none exhibited chloride, calcium and magnesium concentrations above the EU reference values for mineral waters $\left(\mathrm{Cl}^{-}>200 \mathrm{mg} / \mathrm{L}, \mathrm{Ca}^{2+}>150 \mathrm{mg} / \mathrm{L}\right.$ and $\left.\mathrm{Mg}^{2+}>50 \mathrm{mg} / \mathrm{L}\right)$.

\section{Discussion}

\subsection{Groundwater composition and human health}

The Regulation on the Hygienic Acceptability of Potable Water given by the World Health Organization (WHO) defines the Maximum Acceptable Concentration of chemical substances in water for public water supply that corresponds to $1.5 \mathrm{mg} / \mathrm{L}$ for fluoride (WHO, 2011). Five water sources (codes DXE, SL4, SL6, SL7 and SL10) exhibited dissolved $\mathrm{F}^{-}$concentration between 1 and $1.5 \mathrm{mg} / \mathrm{L}$. They are suitable for human consumption (WHO, 2011) and mineral waters according to the EU directive (van der Aa, 2003). The WHO (2011) guideline reference value of $1.5 \mathrm{mg} / \mathrm{L}$ for fluoride has been exceeded in 19 water sources (25.3\%) that are located at Águas de São Pedro (SP), Águas da Prata (SP), Poços de Caldas (MG), Pocinhos do Rio Verde (MG), São Lourenço (MG), Caxambu (MG), and Araxá (MG) spas. Such number is higher than $16 \%$ as reported by Eupedia (2016) for the analyses of different mineral water brands. It requires effective actions from the 
Table 4

Chemical data of the waters analyzed in this study. All results in mg/L.

\begin{tabular}{|c|c|c|c|c|c|c|c|c|c|c|c|c|}
\hline Sample code & $\mathrm{Na}^{+}$ & $\mathrm{K}^{+}$ & $\mathrm{Ca}^{2+}$ & $\mathrm{Mg}^{2+}$ & $\mathrm{HCO}_{3}^{-}$ & $\mathrm{CO}_{3}^{2-}$ & $\mathrm{OH}^{-}$ & $\mathrm{Cl}^{-}$ & $\mathrm{F}^{-}$ & $\mathrm{NO}_{3}^{-}$ & $\mathrm{SO}_{4}^{2-}$ & $\mathrm{PO}_{4}^{3-}$ \\
\hline ALS & 26.9 & 5.38 & 0.29 & 0.45 & 261 & 0 & 0 & 11.6 & 0.07 & 1.4 & 1 & 0.19 \\
\hline GIO & 459.0 & 1.90 & 0.13 & 0.82 & 204 & 0 & 0 & 27.0 & 7.06 & 0.9 & 225 & 0.06 \\
\hline JUV & 598.0 & 1.16 & 1.11 & 0.08 & 338 & 0 & 0 & 28.4 & 8.30 & 11.2 & 126 & 0.08 \\
\hline PLA & 200.0 & 3.99 & 0.04 & 0.98 & 472 & 0 & 0 & 10.2 & 19.71 & 8.5 & 37 & 0.22 \\
\hline POL & 532.0 & 9.84 & 0.02 & 1.00 & 1390 & 0 & 0 & 16.9 & 32.37 & 1.2 & 90 & 0.15 \\
\hline VIT & 567.0 & 13.11 & 0.89 & 0.93 & 1134 & 254 & 0 & 23.5 & 18.36 & 6.6 & 111 & 0.09 \\
\hline BOI & 1.9 & 8.28 & 0.06 & 0.74 & 17 & 0 & 0 & 24.5 & 0.26 & 1.3 & 11 & 0.05 \\
\hline PTA & 5.3 & 4.58 & 0.06 & 0.64 & 73 & 0 & 0 & 5.9 & 0.41 & 1.4 & 8 & 0.40 \\
\hline VIL & 0.6 & 3.44 & 0.62 & 0.24 & 2 & 0 & 0 & 2.9 & 0.08 & 7.8 & 2 & 0.04 \\
\hline PDE & 5.0 & 5.35 & 3.12 & 0.44 & 99 & 0 & 0 & 3.0 & 0.50 & 1.5 & 3 & 0.17 \\
\hline SIL & 3.1 & 2.56 & 0.09 & 0.90 & 36 & 0 & 0 & 3.2 & 0.12 & 2.3 & 1 & 0.10 \\
\hline FIL & 2.3 & 3.20 & 0.84 & 0.75 & 65 & 0 & 0 & 2.2 & 0.12 & 2.6 & 1 & 0.07 \\
\hline BEL & 1.9 & 2.24 & 0.07 & 0.62 & 69 & 0 & 0 & 2.3 & 0.11 & 2.2 & 1 & 0.13 \\
\hline SRE & 1.8 & 4.36 & 0.03 & 0.60 & 58 & 0 & 0 & 2.9 & 0.12 & 1.2 & 1 & 0.29 \\
\hline $\mathrm{COM}$ & 0.7 & 0.65 & 0.21 & 0.31 & 11 & 0 & 0 & 2.5 & 0.09 & 1.4 & 3 & 0.02 \\
\hline LIN & 15.7 & 3.96 & 0.02 & 0.60 & 76 & 0 & 0 & 9.3 & 0.36 & 8.7 & 11 & 0.51 \\
\hline CUR & 1.3 & 3.66 & 3.33 & 0.52 & 60 & 0 & 0 & 2.3 & 0.09 & 1.3 & b.d. & 0.11 \\
\hline SJO & 4.8 & 1.03 & 3.36 & 0.68 & 16 & 0 & 0 & 8.8 & 0.10 & 4.0 & 1 & 0.13 \\
\hline SCA & 5.7 & 2.96 & 3.06 & 0.35 & 71 & 0 & 0 & 3.8 & 0.11 & 3.4 & b.d. & 0.33 \\
\hline ITA & 5.6 & 2.78 & 3.18 & 0.49 & 62 & 0 & 0 & 3.6 & 0.11 & 2.9 & 1 & 0.17 \\
\hline SLU & 5.9 & 1.15 & 3.24 & 0.64 & 35 & 0 & 0 & 4.6 & 0.07 & 1.9 & b.d. & 0.11 \\
\hline SAT & 5.9 & 1.33 & 3.30 & 0.60 & 43 & 0 & 0 & 7.8 & 0.10 & 2.4 & b.d. & 0.16 \\
\hline BRU & 7.2 & 1.84 & 3.48 & 0.62 & 27 & 0 & 0 & 5.8 & 0.13 & 4.7 & b.d. & 0.25 \\
\hline LAN & 5.6 & 0.94 & 3.45 & 0.66 & 21 & 0 & 0 & 8.3 & 0.07 & 4.4 & b.d. & 0.04 \\
\hline SAA & 6.8 & 2.89 & 3.09 & 0.64 & 52 & 0 & 0 & 3.7 & 0.10 & 2.0 & b.d. & 0.22 \\
\hline SBE & 8.2 & 3.72 & 3.00 & 0.43 & 83 & 0 & 0 & 7.8 & 0.10 & 3.9 & 1 & 0.15 \\
\hline BIO & 2.6 & 4.40 & 3.09 & 0.59 & 24 & 0 & 0 & 2.6 & 0.07 & 2.0 & b.d. & 0.09 \\
\hline JOR & 87.8 & 0.42 & 2.31 & b.d. & 0 & 82 & 17 & 7.8 & 0.44 & 1.5 & 78 & 0.12 \\
\hline ADB & 87.4 & 0.36 & 2.46 & 0.01 & 0 & 106 & 24 & 7.0 & 0.40 & 2.6 & 52 & 0.16 \\
\hline CGO & 91.4 & 0.39 & 2.67 & 0.01 & 0 & 52 & 46 & 7.9 & 0.43 & 1.7 & 67 & 0.27 \\
\hline SRC & 91.8 & 0.33 & 2.49 & 0.01 & 0 & 22 & 68 & 7.7 & 0.48 & 1.2 & 74 & 0.22 \\
\hline SEI & 83.5 & 0.29 & 2.40 & 0.01 & 0 & 58 & 47 & 7.0 & 0.49 & 2.9 & 63 & 0.06 \\
\hline BMU & 7.3 & 3.07 & 25.50 & 0.90 & 72 & 1 & 0 & b.d. & 0.02 & 0.6 & b.d. & b.d. \\
\hline LA1 & 4.5 & 5.60 & 3.42 & 0.65 & 25 & 0 & 0 & 5.9 & 0.10 & 3.3 & 1.0 & 0.04 \\
\hline LA2 & 4.3 & 4.95 & 3.39 & 0.65 & 19 & 0 & 0 & 8.0 & 0.10 & 3.1 & 2.0 & 0.06 \\
\hline LA3 & 6.3 & 4.48 & 3.48 & 0.62 & 28 & 0 & 0 & 6.7 & 0.08 & 3.6 & b.d. & 0.05 \\
\hline LA4 & 6.1 & 2.75 & 3,42 & 0.68 & 34 & 0 & 0 & 16.2 & 0.07 & 3.6 & b.d. & 0.02 \\
\hline LA5 & 2.5 & 5.24 & 3.48 & 0.75 & 22 & 0 & 0 & 3.4 & 0.32 & 2.2 & 1 & 0.09 \\
\hline LA6 6 & 2.5 & 4.40 & 3.39 & 0.78 & 21 & 0 & 0 & 3.4 & 0.08 & 1.8 & 1 & 0.04 \\
\hline SL7 & 23.4 & 19.84 & 3.39 & 0.37 & 158 & 0 & 0 & 4.6 & 1.18 & 1.0 & 2 & 0.23 \\
\hline SL5 & 48.5 & 20.10 & 2.94 & 0.06 & 262 & 0 & 0 & 5.4 & 0.59 & 1.8 & b.d. & 0.10 \\
\hline SL6 & 54.3 & 18.05 & 3.18 & 0.30 & 292 & 0 & 0 & 6.1 & 1.17 & 0.4 & 1 & 0.16 \\
\hline SL3 & 46.2 & 11.50 & 3.45 & 0.25 & 303 & 0 & 0 & 3.7 & 1.75 & 0.6 & 1 & 0.09 \\
\hline SL4 & 25.5 & 19.96 & 3.69 & 0.36 & 93 & 0 & 0 & 12.8 & 1.24 & 0.8 & b.d. & 0.06 \\
\hline SL1 & 33.7 & 17.24 & 3.42 & 0.17 & 163 & 0 & 0 & 12.8 & 0.15 & 1.1 & 3 & 0.09 \\
\hline SL10 & 85.6 & 23.85 & 3.27 & 0.12 & 495 & 0 & 0 & 3.4 & 1.07 & 5.9 & b.d. & 0.27 \\
\hline SL9 & 35.2 & 17.52 & 3.48 & 0.39 & 162 & 0 & 0 & 8.0 & 0.89 & b.d. & 3 & 0.07 \\
\hline ROR & 2.6 & 1.92 & 3.39 & 0.68 & 2 & 0 & 0 & 1.7 & 0.11 & 1.6 & b.d. & 0.05 \\
\hline REW & 2.8 & 0.73 & 3.39 & 0.45 & 4 & 0 & 0 & 2.0 & 0.04 & 1.6 & b.d. & 0.09 \\
\hline CAF & 2.3 & 0.90 & 3.30 & 0.58 & 1 & 0 & 0 & 2.0 & 0.08 & 1.2 & b.d. & 0.17 \\
\hline FEP & 12.8 & 6.60 & 3.87 & 0.73 & 50 & 0 & 0 & 3.7 & 0.60 & 1.0 & b.d. & 0.26 \\
\hline MAR & 14.9 & 13.05 & 3.33 & 0.50 & 130 & 0 & 0 & 2.5 & 0.53 & 0.8 & 4 & 0.25 \\
\hline SLI & 2.9 & 1.92 & 3.27 & 0.70 & 31 & 0 & 0 & 1.9 & 0.22 & 1.5 & 1 & 0.05 \\
\hline GFL & 92.3 & 21.10 & 3.42 & 0.17 & 919 & 0 & 0 & 7.1 & 2.02 & 12.3 & 2 & 0.37 \\
\hline VEN & 85.6 & 20.85 & 4.50 & 0.06 & 784 & 0 & 0 & 9.7 & 1.71 & 6.4 & 2 & 0.15 \\
\hline MAY & 6.8 & 12.06 & 3.42 & 0.64 & 75 & 0 & 0 & 3.2 & 0.23 & 1.8 & 1 & 0.11 \\
\hline EGU & 86.2 & 21.35 & 4.50 & 0.35 & 755 & 0 & 0 & 4.6 & 2.25 & 12.5 & b.d. & 0.13 \\
\hline VIO & 9.6 & 15.48 & 3.81 & 0.57 & 130 & 0 & 0 & 2.5 & 0.33 & 1.3 & 1 & 0.13 \\
\hline DPE & 18.6 & 16.48 & 3.42 & 0.51 & 153 & 0 & 0 & 2.4 & 0.42 & 1.5 & 1 & 0.14 \\
\hline $\mathrm{BZA}$ & 108.8 & 25.25 & 4.95 & 0.37 & 680 & 0 & 0 & 5.6 & 2.31 & 18.1 & 4 & 0.12 \\
\hline DXE & 75.1 & 21.50 & 3.99 & 0.01 & 643 & 0 & 0 & 3.3 & 1.34 & 3.8 & b.d. & 0.08 \\
\hline LEO & 35.3 & 5.75 & 3.57 & 0.22 & 253 & 0 & 0 & 6.5 & 0.42 & 1.1 & b.d. & 0.25 \\
\hline ISA & 67.2 & 23.20 & 4.05 & 0.19 & 560 & 0 & 0 & 3.8 & 1.83 & 1.5 & 1 & 0.21 \\
\hline QUI & 10.4 & 8.94 & 1.03 & 0.33 & 10 & 0 & 0 & 11.0 & 0.07 & 7.6 & b.d. & 0.06 \\
\hline NOV & 112.9 & 6.88 & 0.61 & 0.02 & 70 & 126 & 0 & 4.6 & 13.57 & 11.1 & 41 & 0.08 \\
\hline MAC & 183.0 & 10.36 & 0.51 & 0.03 & 0 & 150 & 83 & 6.0 & 22.24 & 3.3 & 76 & 0.14 \\
\hline SIN & 180.0 & 9.58 & 0.69 & 0.03 & 31 & 230 & 0 & 5.8 & 23.00 & 1.0 & 73 & 0.09 \\
\hline FRA & 1.2 & 0.74 & 0.47 & 0.05 & 6 & 0 & 0 & 2.3 & 0.08 & 11.3 & b.d. & 0.02 \\
\hline PEB & 183.0 & 11.12 & 0.68 & 0.04 & 4 & 248 & 0 & 5.7 & 22.20 & 1.9 & 75 & 0.13 \\
\hline RIV & 182.0 & 9.33 & 2.13 & 0.01 & 86 & 184 & 0 & 7.9 & 25.24 & 1.0 & 76 & 0.16 \\
\hline SMA & 182.7 & 10.47 & 2.28 & 0.02 & 123 & 168 & 0 & 7.5 & 26.36 & 1.0 & 78 & 0.05 \\
\hline SJO & 178.0 & 10.62 & 2.43 & 0.02 & 142 & 176 & 0 & 7.3 & 26.05 & 1.3 & 78 & 0.07 \\
\hline AMO & 0.4 & 1.68 & 2.43 & 0.03 & 5 & 0 & 0 & 2.0 & 0.07 & 1.9 & b.d. & 0.11 \\
\hline DBJ & 2.1 & 8.84 & 0.17 & 0.32 & 112 & 0 & 0 & 2.1 & 0.29 & 5.3 & 3 & 0.29 \\
\hline AJU & 1510.0 & 14.91 & 0.42 & 0.11 & 52 & 2160 & 0 & 48.0 & 9.00 & 2.3 & 189 & 1.36 \\
\hline
\end{tabular}

b.d. = below detection limit. 
Table 5

Statistical parameters of the waters analyzed in this study.

\begin{tabular}{|c|c|c|c|c|c|c|}
\hline Parameter & Unit & Minimum & $\begin{array}{l}\text { Lower } \\
\text { quartile }\end{array}$ & Median & $\begin{array}{l}\text { Upper } \\
\text { quartile }\end{array}$ & Maximum \\
\hline Temp. & ${ }^{\circ} \mathrm{C}$ & 20.4 & 23.4 & 24.6 & 25.8 & 35.7 \\
\hline $\mathrm{pH}$ & - & 4.16 & 5.86 & 6.41 & 7.94 & 9.60 \\
\hline Eh & $\mathrm{mV}$ & -159 & -72 & -52 & -28 & 112 \\
\hline $\mathrm{EC}$ & $\mu \mathrm{S} / \mathrm{cm}$ & 20 & 170 & 360 & 1235 & 6390 \\
\hline DO & $\mathrm{mg} / \mathrm{L}$ & 0.78 & 1.78 & 3.13 & 4.77 & 9.05 \\
\hline $\mathrm{CO}_{2}$ & $\mathrm{mg} / \mathrm{L}$ & 0 & 110 & 236 & 1056 & 1840 \\
\hline $\mathrm{H}_{2} \mathrm{~S}$ & $\mu \mathrm{g} / \mathrm{L}$ & $<1$ & 2 & 4 & 13 & 3064 \\
\hline ALK & $\mathrm{mg} / \mathrm{L}$ & 1 & 32 & 93 & 261 & 2212 \\
\hline TDS & $\mathrm{mg} / \mathrm{L}$ & 11 & 135 & 229 & 459 & 2898 \\
\hline IS & $\times 10^{-4}$ & 3.1 & 8.5 & 19.9 & 67.5 & 793.6 \\
\hline $\mathrm{SiO}_{2}$ & $\mathrm{mg} / \mathrm{L}$ & 6.7 & 17.8 & 28.3 & 34.5 & 63.5 \\
\hline $\mathrm{Fe}_{\text {tot }}$ & $\mathrm{mg} / \mathrm{L}$ & $<0.006$ & 0.01 & 0.04 & 0.14 & 5.18 \\
\hline $\mathrm{Na}^{+}$ & $\mathrm{mg} / \mathrm{L}$ & 0.4 & 4.4 & 12.8 & 86.8 & 1510.0 \\
\hline $\mathrm{K}^{+}$ & $\mathrm{mg} / \mathrm{L}$ & 0.29 & 2.08 & 4.95 & 11.78 & 25.25 \\
\hline $\mathrm{Ca}^{2+}$ & $\mathrm{mg} / \mathrm{L}$ & 0.02 & 0.76 & 3.18 & 3.42 & 25.50 \\
\hline $\mathrm{Mg}^{2+}$ & $\mathrm{mg} / \mathrm{L}$ & $<0.006$ & 1.00 & 0.12 & 0.45 & 0.64 \\
\hline $\mathrm{Cl}^{-}$ & $\mathrm{mg} / \mathrm{L}$ & $<0.3$ & 48.0 & 3.1 & 5.6 & 7.8 \\
\hline $\mathrm{F}^{-}$ & $\mathrm{mg} / \mathrm{L}$ & 0.02 & 0.10 & 0.40 & 1.73 & 32.27 \\
\hline $\mathrm{NO}_{3}^{-}$ & $\mathrm{mg} / \mathrm{L}$ & $<0.8$ & 1.3 & 1.9 & 3.6 & 18.1 \\
\hline $\mathrm{SO}_{4}^{2-}$ & $\mathrm{mg} / \mathrm{L}$ & $<0.5$ & 1 & 1 & 24 & 225 \\
\hline $\mathrm{PO}_{4}^{3-}$ & $\mathrm{mg} / \mathrm{L}$ & $<0.02$ & 0.07 & 0.12 & 0.18 & 1.36 \\
\hline
\end{tabular}

$\mathrm{EC}=$ electrical conductivity; $\mathrm{DO}=$ dissolved oxygen; $\mathrm{TDS}=$ total dissolved solids; $\mathrm{ALK}=$ total alkalinity; IS = ionic strength.

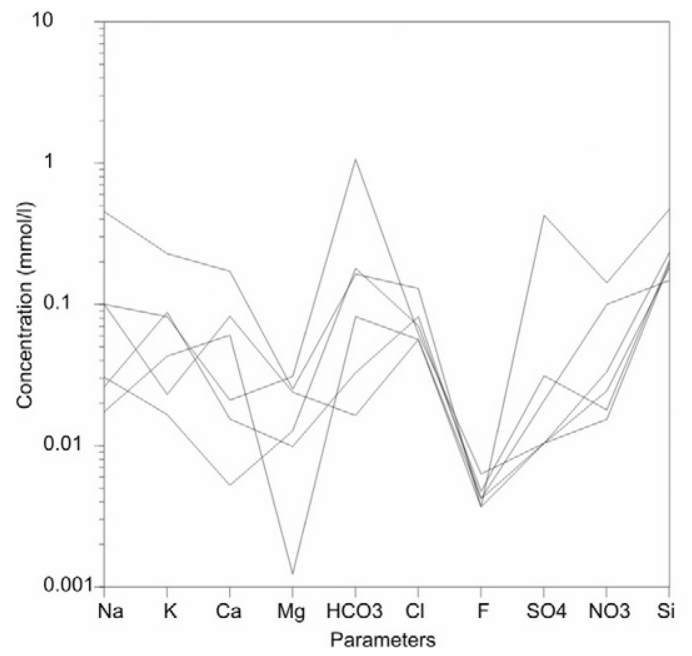

national health agencies as dental fluorosis may develop from successive exposures to high $\mathrm{F}^{-}$concentrations during the water ingestion. This disease is a developmental disturbance leading to dental enamel with lower mineral content and increased porosity (Alvarez et al., 2009; Edmunds and Smedley, 2013).

The high dissolved $\mathrm{SiO}_{2}$ levels in the water sources analyzed may imply on the health treatment. Davenward et al. (2013) used Si-rich mineral waters as non-invasive methods to reduce the body burden of aluminum in individuals with Alzheimer's disease and a control group (their carers and partners). Davenward et al. (2013) reported that drinking Si-rich mineral water ( 1 L each day for 12 weeks) facilitated the $\mathrm{Al}$ removal via the urine in both patient and control groups without any concomitant effect upon the urinary excretion of the essential metals (Fe and Cu). Davenward et al. (2013) utilized the Malaysian mineral water "Spritzer" ( $35 \mathrm{mg} / \mathrm{L}$ of $\mathrm{Si}$ ) in their experiments, suggesting other brands on sale in Britain with high Si levels ("Volvic"-20 mg/L; "Fiji"-45 mg/L). The highest dissolved $\mathrm{SiO}_{2}$ concentration was $63.5 \mathrm{mg} / \mathrm{L}$ (Si concentration $30 \mathrm{mg} / \mathrm{L}$, code ISA in Table 3), almost the same level used by Davenward et al. (2013). This spring name remembers the visit of Isabel Princess and her husband to Caxambu spa in 1868 for her health treatment (infertility due to a profound anemia). She was cured after 1-month treatment with those waters also Fe-enriched (3.86 mg/L, Table 3).

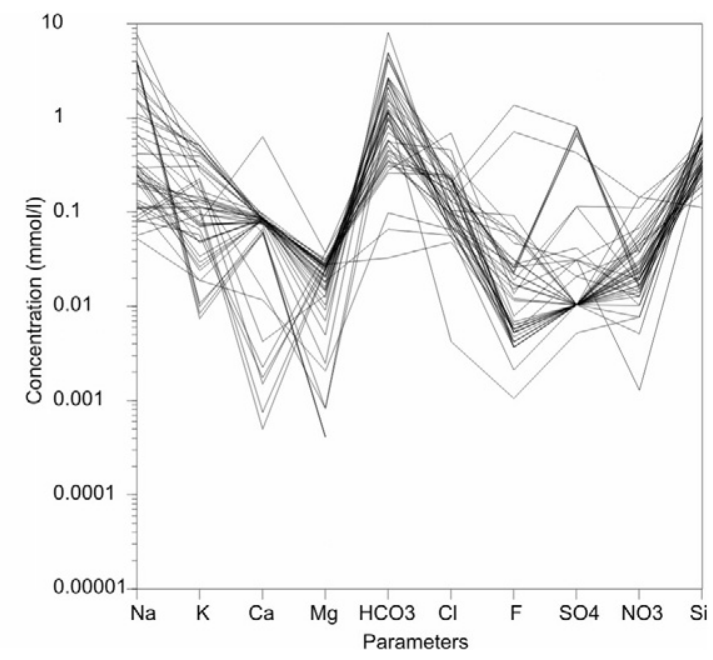

Fig. 2. Schoeller (1962) diagrams for the waters containing very low mineral concentration (TDS < $50 \mathrm{mg} / \mathrm{L}$ ) (left) and low mineral concentration (TDS 50-500 mg/L) (right).
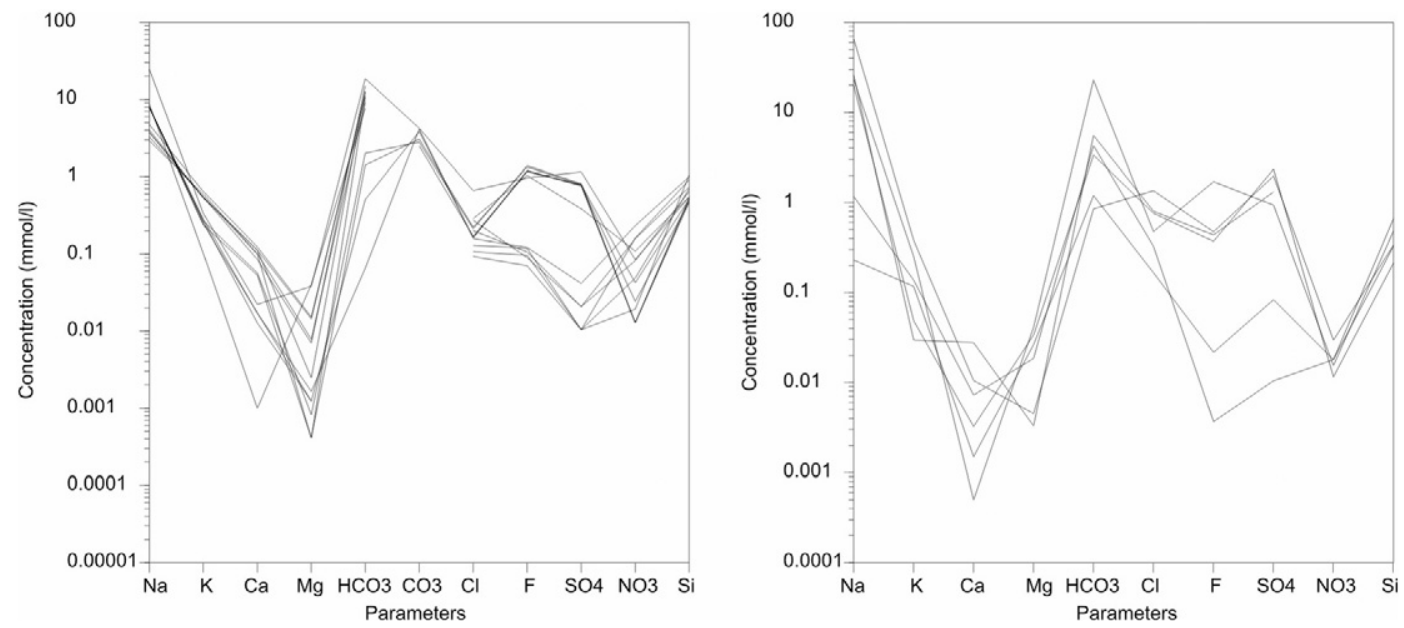

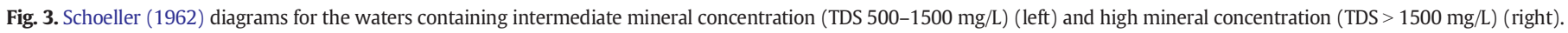




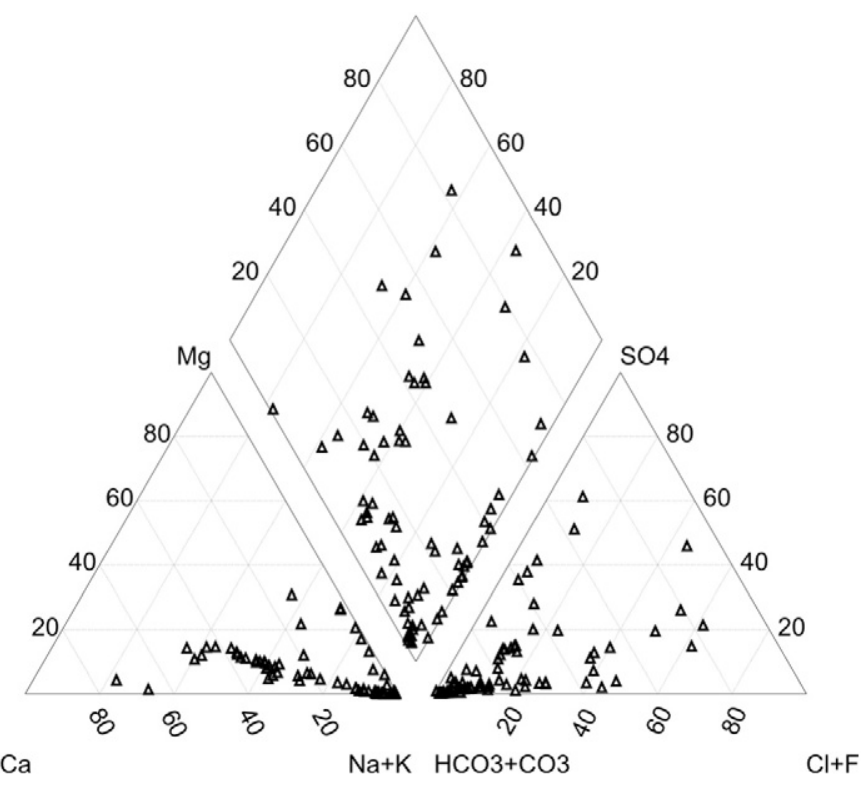

Fig. 4. Data of the groundwaters in this research plotted in a Piper (1944) diagram including the presence of the anion fluoride.

\subsection{Major hydrochemical trends}

The parameters $\mathrm{pH}$ and Eh reflect, respectively, the protons and electrons activities in the environment. The use of Eh-pH diagrams in geochemistry increased since the pioneering studies of Garrels (1959); Baas Becking et al. (1960) and Garrels and Christ (1965). Appropriate thermodynamic data have been utilized for many reactions occurring in temperature and pressure conditions different of those of the standard state $\left(25^{\circ} \mathrm{C}\right.$ and 1 bar), despite the pressure changes do not introduce significant errors in the Eh-pH contours calculated at 1 bar

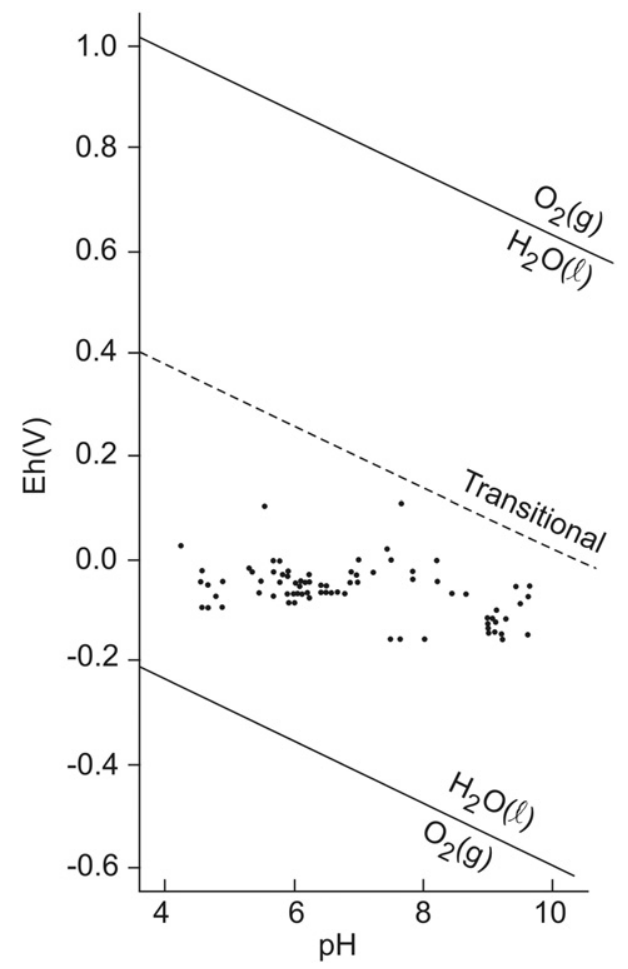

Fig. 5. Data of the groundwaters in this research plotted in an Eh-pH diagram as utilized by Krauskopf and Bird (1995).
(Brookins, 1988). In natural environments, oxidizing acid and reducing basic systems are often reported in the literature (e.g. Baas Becking et al., 1960; Krauskopf and Bird, 1995; Brownlow, 1996). The waters analyzed are reducing in character according to the Eh-pH diagram (Fig. 5).

The $\mathrm{pH}$ and Eh in the water sources may be controlled by the $\mathrm{Fe}(\mathrm{OH})_{3}-\mathrm{Fe}_{2} \mathrm{O}_{3}$ precipitation in the inert electrode as this is a common phenomenon in groundwater containing ferrous iron (Barnes and Back, 1964). Fig. 6 shows the $\mathrm{pH}$ and Eh data plotted in the activity diagram involving the chemical equilibrium between water and the system $\mathrm{FeS}-\mathrm{H}_{2} \mathrm{~S}-\mathrm{H}_{2} \mathrm{SO}_{4}$. Most of the samples are in the field of hematite coexisting with dissolved sulfate, whereas some are within the fields defined by pyrite and $\mathrm{Fe}^{2+}$ coexisting with dissolved sulfate, and pyrite coexisting with sulfur. The generation of sulfate from pyrite oxidation occurs in some sites according to the following reaction (Krauskopf and Bird, 1995):

$2 \mathrm{FeS}_{2}+7.5 \mathrm{O}_{2}+\mathrm{H}_{2} \mathrm{O} \rightarrow 2 \mathrm{Fe}^{3+}+4 \mathrm{SO}_{4}{ }^{2-}+2 \mathrm{H}^{+}$

However, the $\mathrm{pH}$ lowering associated to pyrite oxidation may not happen due to neutralization reactions involving the carbonates dissolution. Speciation calculations done with the Aquachem 4.0 software (Waterloo Hydrogeologic, 2003) yielded positive mineral saturation indices (oversaturation) for hematite (59\%, 44 water sources) and negative values (undersaturation) for pyrite ( $71 \%, 53$ water sources).

TDS and EC are directly related as reported elsewhere (e.g. Hem, 1985) (Fig. 7). Using the TDS-EC relationship, Dinelli et al. (2010) classified the Italian bottled mineral waters according to EC rather than

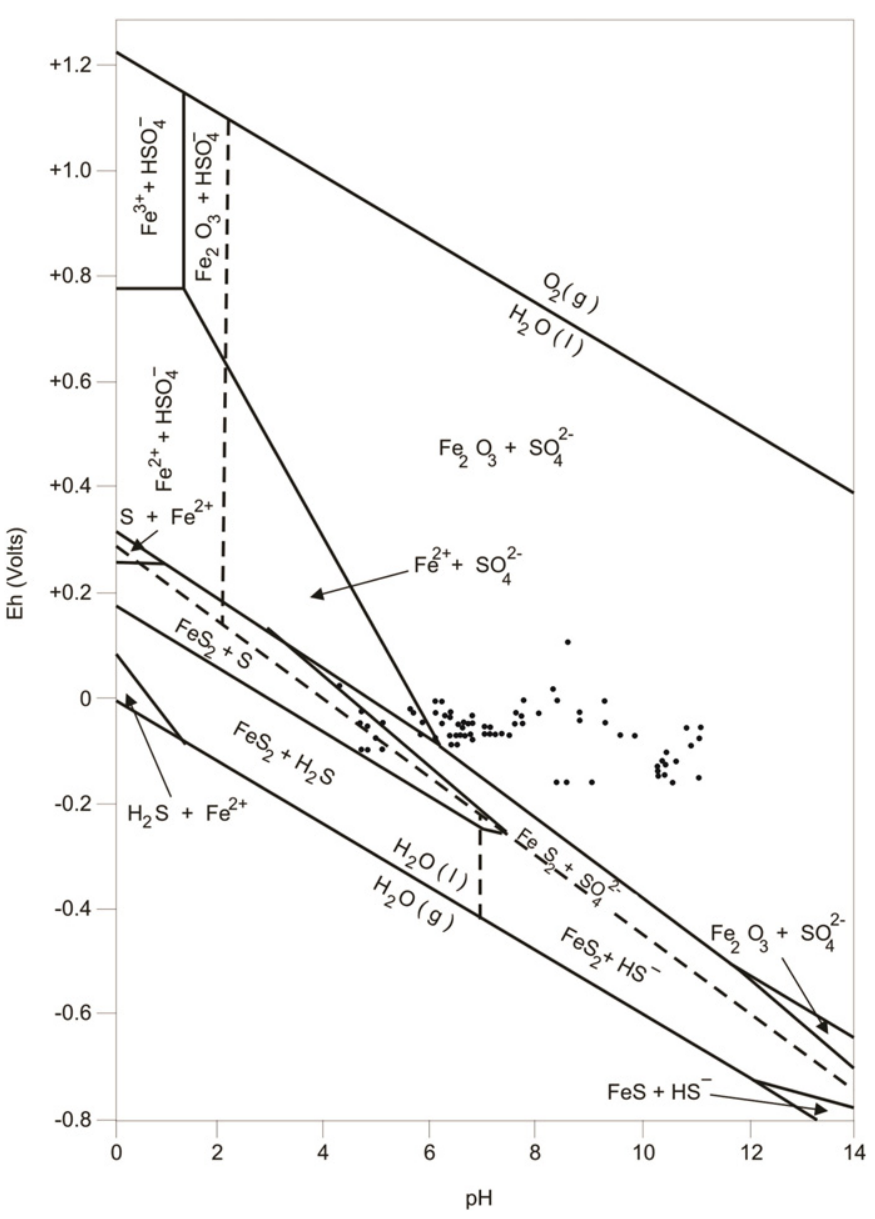

Fig. 6. Groundwater data plotted on Eh-pH diagram of iron oxides and sulfides in the presence of water at $25{ }^{\circ} \mathrm{C}$ temperature and $1 \mathrm{~atm}$ pressure, containing $\Sigma \mathrm{S}=0.1 \mathrm{M}$ (Faure, 1998). The dashed lines indicate the conditions under which the different $S$ species become dominant. 

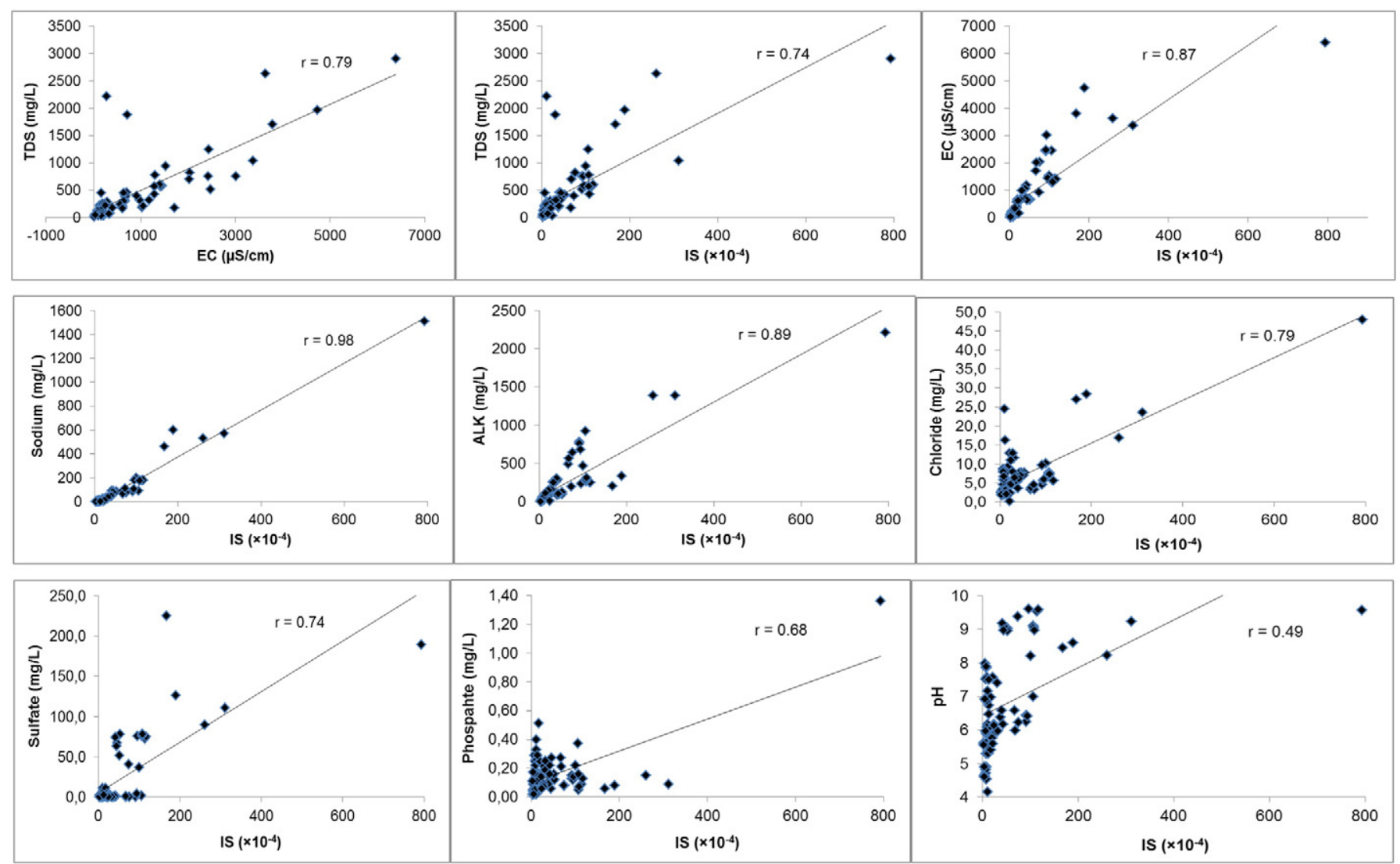

Fig. 7. The TDS-EC relationship and plots of the TDS, EC, sodium, total alkalinity (ALK), chloride, sulfate, phosphate and pH in the groundwater sources versus their ionic strength (IS).

TDS that is the parameter ruled by the EU mineral water directive (van $\operatorname{der} A a, 2003)$. IS and TDS are also related ( $r=0.74$ ) (Fig. 7), implying on a IS-EC relationship (Fig. 7), despite the non-existence of a theoretical relation between them. The ions justifying such trends are sodium, (bi)carbonate, chloride, sulfate and phosphate that are mathematically related with IS (Fig. 7) (Krauskopf and Bird, 1995). Sodium and sulfate correlated with $\mathrm{pH}$, explaining the $\mathrm{pH}-\mathrm{IS}$ relationship (Fig. 7).

The high $\mathrm{SiO}_{2}$ concentration (Figs. 2 and 3 ) is a differential aspect of the spas groundwaters relative to many elsewhere. For instance, the analyses of 36 European mineral water brands reported by Eupedia (2016) show the following scenario for the molar ratios higher than unity: $\mathrm{SiO}_{2} / \mathrm{HCO}_{3}^{-}$and $\mathrm{SiO}_{2} / \mathrm{Ca}^{2+}=1$ mineral water (3\%), $\mathrm{SiO}_{2} /$ $\mathrm{Na}^{+}=2$ mineral waters (6\%), $\mathrm{SiO}_{2} / \mathrm{Mg}^{2+}=3$ mineral waters (8\%), $\mathrm{SiO}_{2} / \mathrm{K}^{+}=11$ mineral waters (30\%). This number is much higher in the spas groundwaters of this research (Tables 3 and 4$): \mathrm{SiO}_{2} /$ $\mathrm{HCO}_{3}^{-}=14$ water sources (19\%), $\mathrm{SiO}_{2} / \mathrm{Na}^{+}=34$ water sources (45\%), $\mathrm{SiO}_{2} / \mathrm{Ca}^{2+}$ and $\mathrm{SiO}_{2} / \mathrm{Mg}^{2+}=75$ water sources $(100 \%), \mathrm{SiO}_{2} / \mathrm{K}^{+}=66$ water sources (88\%).

Thus, several hydrochemical types represent the composition of waters from the different aquifer systems in this research. The great hydrochemical variability suggests that mixing of groundwaters from various sources often occur as recognized by Bonotto (2016) from the U-isotopes modeling. There is no way to obtain a consistent spatial representation of the hydrochemical facies in the whole study area. Very dilute waters suggesting the rainwater composition with small amounts of constituents added within the aquifers are widely spread. Additionally, saline waters also occur in some spas due to extensive chemical interaction between the groundwater and the aquifer materials.

\subsection{Major sources influencing the groundwater composition}

The aquifer systems investigated belong to two principal hydrogeological provinces: Paraná and Southeastern Shield (Mente,
2008). Such classification is general and does not allow discriminate between the shallower and deeper aquifers sometimes sampled in the same spa. This happened at Araxá, São Lourenço, Caxambu and Poços de Caldas spas (MG) where more superficial and deeper zones of water circulation could explain the main differences on the physicochemical parameters (Cruz and Peixoto, 1989). Bertolo et al. (2007) pointed out that the groundwater flow in rapid and shallow $(<70 \mathrm{~m})$ systems would imply in waters with low mineralization, whereas the increase on the amount of dissolved species would tend to occur in deeper systems characterized by waters possessing long residence time. Additional aspects justifying such differences would certainly be the diverse lithology of the research area that comprises various types of magmatic, sedimentary and metamorphic rocks.

Aquachem 4.0 software (Waterloo Hydrogeologic, 2003) does the rock source deduction based on $\mathrm{SiO}_{2}$ (in $\mathrm{mmol} / \mathrm{L}$ ), $\mathrm{TDS}$ (in $\mathrm{mg} / \mathrm{L}$ ), and the following ratios (Hounslow, 1995): $\mathrm{HCO}_{3}^{-} / \mathrm{SiO}_{2}, \quad \mathrm{SiO}_{2} /$ $\left(\mathrm{Na}^{+}+\mathrm{K}^{+}-\mathrm{Cl}^{-}\right),\left(\mathrm{Na}^{+}+\mathrm{K}^{+}-\mathrm{Cl}^{-}\right) /\left(\mathrm{Na}^{+}+\mathrm{K}^{+}-\mathrm{Cl}^{-}+\mathrm{Ca}^{2+}\right)$, $\mathrm{Na}^{+} /\left(\mathrm{Na}^{+}+\mathrm{Cl}^{-}\right), \mathrm{Mg}^{2+} /\left(\mathrm{Ca}^{2+}+\mathrm{Mg}^{2+}\right), \mathrm{Ca}^{2+} /\left(\mathrm{Ca}^{2+}+\mathrm{SO}_{4}^{2-}\right), \mathrm{Cl}^{-} /$ $\sum$ anions, $\mathrm{HCO}_{3}^{-} / \sum$ anions. The aquifer systems in this research are inland, not suffering influence of processes affecting the coastal areas like salinisation due to sea water intrusion. Therefore, ratios evidencing them are not helpful for deducting some possible sources responsible by the presence of dissolved constituents in the liquid phase.

Demetriades (2010) have attributed $\mathrm{Na}^{+} / \mathrm{K}^{+}$ratios $<10$ to rainwater, characterizing them in some bottled waters situated in areas with a comparatively high annual rainfall. In this paper, most of the waters analyzed $\left(76 \%\right.$ ) exhibited $\mathrm{Na}^{+} / \mathrm{K}^{+}$ratios $<10$ but many of them are not indicative of rapid groundwater flow as expected for a rainwater source. The $\mathrm{Na}^{+} /\left(\mathrm{Na}^{+}+\mathrm{Cl}^{-}\right)$ratios $<0.5$ and TDS values $<50 \mathrm{mg} / \mathrm{L}$ suggest a rainwater source according to the software Aquachem 4.0. They were found for the springs VIL (Águas da Prata - SP), COM (Águas de Lindóia - SP) and AMO (Pocinhos do Rio Verde - MG), agreeing with their location, flow conditions, and rainfall regime. 
Demetriades (2010) found $\left(\mathrm{Ca}^{2+}+\mathrm{Mg}^{2+}\right) /\left(\mathrm{Na}^{+}+\mathrm{K}^{+}\right)$ratios $>1.0$ in all bottled waters analyzed which would indicate that the aquifers are subjected to a continuous recharge. Equivalent ratios and processes have been identified from chemical and hydrogeological data of the samples coded BMU and AMO.

Demetriades (2010) evaluated $\mathrm{Cl}^{-} / \sum$ anions $<0.8$ in all bottled waters analyzed and pointed out that they could not be seriously affected by dissolution of halite or other evaporitic minerals. Equivalent ratios were also found in all spas groundwaters, yielding the "rock weathering" source classification by the Aquachem 4.0 software.

The rock source deduction by Aquachem 4.0 software based on the TDS concentration was "silicate weathering" (56 water sources, $75 \%$ ) and "carbonate weathering" (19 water sources, $25 \%$ ). The deduction from the $\mathrm{HCO}_{3}^{-} / \mathrm{SiO}_{2}$ ratio yielded "silicate weathering" (47 water sources, $63 \%$ ), "carbonate weathering" (12 water sources, $16 \%$ ) and mixed "silicate/carbonate weathering" source (16 water sources, $21 \%$ ). These modeled rock sources are compatible with the respective lithologies of the sites.

Fluorite mineralization occurred in different stages of the hydrothermal event in PCAM (Holmes et al., 1992). Saturation indices for fluorite evaluated by the Aquachem 4.0 software indicated oversaturation in the springs RIV, SMA and SJO (Pocinhos do Rio Verde spa) and undersaturation in other groundwater samples. Anthropogenic inputs like the partially treated and untreated sewage, runoff from agricultural sites, and application of some lawn fertilizers cannot justify the dissolved $\mathrm{PO}_{4}^{3-}$ concentration in the spas groundwaters. Apatite occurs in PCAM and other geological contexts (Schorscher and Shea, 1992;
Traversa et al., 2001) and its dissolution may be the most probable $\mathrm{PO}_{4}^{3-}$ source in the water sources. It is a ubiquitous accessory mineral in igneous and metamorphic rocks whose solubility increases with temperature, pressure and raising of the $\mathrm{NaCl}$ mole fraction (Smith et al., 1977; Antignano and Manning, 2008). The highest phosphate concentration $(1.36 \mathrm{mg} / \mathrm{L})$ was in spring AJU at Araxá-MG spa, where a weathering mantle primarily enriched in $\mathrm{P}_{2} \mathrm{O}_{5}, \mathrm{Nb}_{2} \mathrm{O}_{5}, \mathrm{TiO}_{2}, \mathrm{BaO}$ and $\mathrm{REE}_{2} \mathrm{O}_{3}$ and resulting from the alteration of the alkaline-carbonatite rocks has been developed (Traversa et al., 2001). The weathering mantle also contains a large phosphate reserve situated at the northwestern portion of the carbonatitic complex (Traversa et al., 2001).

\subsection{Geothermometry}

The geothermometers use to both natural spring discharges and well fluids provides valuable insight to the nature of the system. Chemical geothermometers depend on the existence at depth of a mineral-fluid equilibrium temperature and its preservation during the fluid passage to the surface. Solute (water) geothermometers were empirically developed or based on thermodynamic properties. The most used are the silica (quartz and chalcedony), $\mathrm{Na} / \mathrm{K}$ and $\mathrm{Na}-\mathrm{K}-\mathrm{Ca}$ geothermometers, also existing others based on $\mathrm{Na} / \mathrm{Li}, \mathrm{Li} / \mathrm{Mg}, \mathrm{K} / \mathrm{Mg}$ ratios and $\mathrm{Na}-\mathrm{K}-\mathrm{Mg}$ relationships (Arnórsson, 2000). The choice of a specific geothermometer must take into account the temperature range for which it is valid as most of them only work above $100{ }^{\circ} \mathrm{C}$ (Arnórsson, 2000).

Geothermometers are generally derived from the Van't Hoff equation, allowing estimate the original subsurface reservoir temperature

Table 6

Geotemperatures (in ${ }^{\circ} \mathrm{C}$ ) estimated according to the equations proposed by Fournier (1977) ( $\mathrm{SiO}_{2}$ geothermometer) and Arnórsson et al. (1983) (Na/K geothermometer).

\begin{tabular}{|c|c|c|c|c|c|}
\hline Sample code & $\mathrm{SiO}_{2}$ geotemperature & $\mathrm{Na} / \mathrm{K}$ geotemperature & Sample code & $\mathrm{SiO}_{2}$ geotemperature & $\mathrm{Na} / \mathrm{K}$ geotemperature \\
\hline ALS & 91 & 278 & LA6 & 52 & 975 \\
\hline GIO & 47 & 3 & SL7 & 86 & 603 \\
\hline JUV & 71 & -21 & SL5 & 69 & 405 \\
\hline PLA & 84 & 73 & SL6 & 93 & 361 \\
\hline POL & 78 & 69 & SL3 & 90 & 311 \\
\hline VIT & 83 & 82 & SL4 & 65 & 576 \\
\hline BOI & 61 & 2364 & SL1 & 59 & 453 \\
\hline PTA & 63 & 610 & SL10 & 85 & 330 \\
\hline VIL & 36 & 3704 & SL9 & 66 & 448 \\
\hline PDE & 62 & 695 & ROR & 61 & 556 \\
\hline SIL & 57 & 594 & REW & 54 & 318 \\
\hline FIL & 51 & 825 & CAF & 44 & 393 \\
\hline BEL & 58 & 739 & FEP & 112 & 455 \\
\hline SRE & 54 & 1259 & MAR & 111 & 615 \\
\hline $\mathrm{COM}$ & 46 & 637 & SLI & 56 & 523 \\
\hline LIN & 89 & 313 & GFL & 96 & 298 \\
\hline CUR & 59 & 1444 & VEN & 90 & 308 \\
\hline SJO & 60 & 288 & MAY & 65 & 981 \\
\hline SCA & 85 & 457 & EGU & 106 & 310 \\
\hline ITA & 85 & 446 & VIO & 69 & 915 \\
\hline SLU & 72 & 275 & DPE & 66 & 619 \\
\hline SAT & 78 & 296 & BZA & 111 & 300 \\
\hline BRU & 83 & 315 & DXE & 94 & 334 \\
\hline LAN & 64 & 254 & LEO & 95 & 251 \\
\hline SAA & 86 & 410 & ISA & 113 & 368 \\
\hline SBE & 92 & 425 & QUI & 39 & 608 \\
\hline BIO & 64 & 947 & NOV & 77 & 149 \\
\hline JOR & 85 & 8 & MAC & 81 & 143 \\
\hline ADB & 83 & 3 & SIN & 82 & 138 \\
\hline CGO & 85 & 4 & FRA & 27 & 502 \\
\hline SRC & 84 & -2 & PEB & 77 & 149 \\
\hline SEI & 89 & -3 & RIV & 80 & 135 \\
\hline BMU & 91 & 408 & SMA & 77 & 144 \\
\hline LA1 & 44 & 766 & SJO & 78 & 148 \\
\hline LA2 & 47 & 728 & AMO & 42 & 2250 \\
\hline LA3 & 44 & 544 & DBJ & 69 & 2257 \\
\hline LA4 & 38 & 424 & AJU & 64 & 38 \\
\hline LA5 & 57 & 1116 & & & \\
\hline
\end{tabular}


Table 7

Subsurface temperatures (in ${ }^{\circ} \mathrm{C}$ ) estimated by the silica and $\mathrm{Na} / \mathrm{K}$ geothermometers.

\begin{tabular}{|c|c|c|c|c|}
\hline \multirow[t]{2}{*}{ Sample code } & \multicolumn{2}{|c|}{ Silica geothermometer } & \multicolumn{2}{|c|}{$\mathrm{Na} / \mathrm{K}$ geothermometer } \\
\hline & Ritter (2012) & This study & Ritter (2012) & This study \\
\hline MAC & $76-92$ & 81 & $89-148$ & 143 \\
\hline PEB & 77-93 & 77 & $90-149$ & 149 \\
\hline NOV & $74-91$ & 77 & $84-144$ & 149 \\
\hline
\end{tabular}

(T) of ascending groundwater which underwent conductive cooling during its ascent. The equation has the form (Waterloo Hydrogeologic, 2003):

$\mathrm{T}(\stackrel{\AA}{C})=[a /(b+\log K)]+273.15$

where: $a$ and $b$ are constants and $K$ is the equilibrium constant that depends on the reaction used for the geothermometer.

The mineral-solution reaction using quartz (silica-geothermometer) is: $\mathrm{SiO}_{2 \text { (qtz) }}+2 \mathrm{H}_{2} \mathrm{O}=\mathrm{H}_{4} \mathrm{SiO}_{4}^{0}$. The cation exchange reaction between albite and K-feldspar depends on the temperature, supporting the $\mathrm{Na} / \mathrm{K}$ geothermometer (Verma et al., 2008). Both feldspars are highly ordered with respect to $\mathrm{Al}$ and $\mathrm{Si}$ (low albite and microcline) and the reaction involved is (Arnórsson, 2000): $\mathrm{NaAl}_{3} \mathrm{SiO}_{8}+\mathrm{K}^{+}=\mathrm{KAl}_{3} \mathrm{SiO}_{8}+\mathrm{Na}^{+}$.

The database in this research allowed estimate the silica and $\mathrm{Na} / \mathrm{K}$ geotemperatures (Table 6) from the following equations $\left(25^{\circ} \mathrm{C}-250^{\circ}\right.$ C) given by Fournier (1977) and Arnórsson et al. (1983), respectively:

$\mathrm{T}(\AA)=[1309 /(5.19-\log S)]-273.15$

$\mathrm{T}(\stackrel{\AA}{C})=[933 /(0.993+\log S P)]-273.15$

where: $S$ represents the dissolved $\mathrm{SiO}_{2}$ concentration (in $\mathrm{mg} / \mathrm{L}$ ) and $\mathrm{SP}$ represents the $\mathrm{Na} / \mathrm{K}$ ratio calculated from the dissolved $\mathrm{Na}$ and $\mathrm{K}$ contents (in $\mathrm{mg} / \mathrm{L}$ ).

Table 7 shows the geotemperatures for the springs MAC, PEB and NOV (Poços de Caldas-MG spa) estimated from Eqs. (3) and (4) and by Ritter (2012) from the SolGeo software (Verma et al., 2008). They practically agree for both geothermometers, inclusive in terms of the higher $\mathrm{Na} / \mathrm{K}$ geotemperatures relative to the silica geotemperatures. Ritter (2012) pointed out that the missing quartz mineral phase in the host rock mineralogy of PCAM would make doubtful the application of the silica geothermometer there. However, most of the $\mathrm{Na} / \mathrm{K}$ geotemperatures ranging from $-21{ }^{\circ} \mathrm{C}$ (JUV spring) to $3704{ }^{\circ} \mathrm{C}$ (VIL spring) (Table 6) are impossible to occur in the aquifer systems investigated. Contrarily, the silica geotemperatures between $27^{\circ} \mathrm{C}$ and $113^{\circ} \mathrm{C}$ (Table 6) are possible in the old low-enthalpy and non-active volcanic systems investigated that differ of those described by Fournier (1977) and Arnórsson et al. (1983). In the case of PCAM, despite quartz is absent in the nepheline-syenites and phonolites, their $\mathrm{SiO}_{2}$ concentration is 52.6\%-55.6\% (Schorscher and Shea, 1992). Thus, such $\mathrm{SiO}_{2}$ levels favor its transfer into the liquid phase when the ascending waters interact with the aquifers rocks, justifying the silica geothermometer use.

\section{Conclusion}

Spas groundwaters from São Paulo (SP) and Minas Gerais (MG) states, Brazil, are an important resource extensively used for drinking in public places, bottling and bathing purposes, among others. This investigation involved the sampling 75 groundwaters from springs and tube wells occurring in 14 municipalities. The Brazilian Code of Mineral Waters (BCMW) guidelines indicated that most of them are cold $\left(<25^{\circ}\right.$ C), $44 \%$ are hypothermal $\left(25-33^{\circ} \mathrm{C}\right)$, one is mesothermal $\left(33-36{ }^{\circ} \mathrm{C}\right)$, three are sulfured and one is ferruginous. The mineral waters EU directive allowed classify them as containing very low mineral concentration (TDS $<50 \mathrm{mg} / \mathrm{L}, 6$ samples), low mineral concentration (TDS 50-
$500 \mathrm{mg} / \mathrm{L}, 50$ samples), intermediate mineral concentration (TDS 500$1500 \mathrm{mg} / \mathrm{L}, 13$ samples), high mineral concentration (TDS > $1500 \mathrm{mg} /$ $\mathrm{L}, 6$ samples), bicarbonate $\left(\mathrm{HCO}_{3}^{-}>600 \mathrm{mg} / \mathrm{L}-7\right.$ samples $)$, sulfate $\left(\mathrm{SO}_{4}^{2-}>200 \mathrm{mg} / \mathrm{L}-1\right.$ sample), fluoride ( $\mathrm{F}^{-}>1 \mathrm{mg} / \mathrm{L}-24$ samples), iron ( $\mathrm{Fe}^{2+}>1 \mathrm{mg} / \mathrm{L}-3$ samples), acidity $\left(\mathrm{CO}_{2}>250 \mathrm{mg} / \mathrm{L}-36 \mathrm{sam}-\right.$ ples), and sodium $\left(\mathrm{Na}^{+}>200 \mathrm{mg} / \mathrm{L}-6\right.$ samples, $8 \%$ ), whilst none exhibited chloride $\left(\mathrm{Cl}^{-}>200 \mathrm{mg} / \mathrm{L}\right)$, calcium $\left(\mathrm{Ca}^{2+}>150 \mathrm{mg} / \mathrm{L}\right)$ and magnesium $\left(\mathrm{Mg}^{2+}>50 \mathrm{mg} / \mathrm{L}\right)$. Fluoride exceeded the WHO maximum allowable for ingestion in waters $(1.5 \mathrm{mg} / \mathrm{L})$ in 19 samples taken from two spas in SP and five spas in MG. The results of the data treatment and plotting according to Aquachem 4.0 software showed the occurrence of various hydrochemical facies, most of them dominated by the presence of $\mathrm{HCO}_{3}^{-}\left(\mathrm{CO}_{3}^{2-}\right)$ and $\mathrm{Na}^{+}$. Additionally, silica was a significant constituent of all waters analyzed unlike most of European bottled mineral waters. The rock source deduction by Aquachem 4.0 software and based on $\mathrm{Cl}^{-} / \sum$ anions ratios $<0.8$ in all water sources indicated the "rock weathering" source classification, whilst the TDS content (in $\mathrm{mg} / \mathrm{L}$ ) and $\mathrm{HCO}_{3}^{-} / \mathrm{SiO}_{2}$ ratios suggested the sources "silicate weathering", "carbonate weathering" and mixed "silicate/carbonate weathering". Testing the silica and $\mathrm{Na} / \mathrm{K}$ geothermometers from the hydrochemical database allowed verify that most of the $\mathrm{Na} / \mathrm{K}$ geotemperatures are impossible to occur in the natural systems, whereas the silica geotemperatures are compatible with the old low-enthalpy and non-active volcanic characteristics of the systems investigated.

\section{Acknowledgments}

The author thanks FAPESP (Foundation for Supporting Research at the State of São Paulo) (Proc. No. 2013/24360-6) and CNPq (National Council for Scientific and Technological Development) (Proc. No. 301462/2011-9), Brazil, for financial support of this investigation. Leila S. Marques and one anonymous reviewer are greatly thanked by helpful comments that improved the readability of the manuscript.

\section{References}

Almeida, F.F.M., Hasui, Y., 1984. O Pré-cambriano do Brasil. Edgard Blücher, São Paulo (378 pp.).

Alvarez, J.A., Rezende, K.M.P.C., Marocho, S.M.S., Alves, F.B.T., Celiberti, P., Ciamponi, A.L., 2009. Dental fluorosis: exposure, prevention and management. J. Clin. Exp. Dent. 1 (1), e14-e18.

Antignano, A., Manning, C.E., 2008. Fluorapatite solubility in $\mathrm{H}_{2} \mathrm{O}$ and $\mathrm{H}_{2} \mathrm{O}-\mathrm{NaCl}$ at 700 to $900{ }^{\circ} \mathrm{C}$ and 0.7 to $2.0 \mathrm{GPa}$. Chem. Geol. 251, 112-119.

APHA (American Public Health Association), 1989. Standard Methods for the Examination of Water and Wastewater. 17th ed. APHA, Washington, DC.

Appelo, C.A.J., Postma, D., 2004. Geochemistry, Groundwater and Pollution. second ed. Balkema, New York.

Arnórsson, S., 2000. Isotopic and Chemical Techniques in Geothermal Exploration, Development and Use: Sampling Methods, Data Handling, Interpretation. IAEA (International Atomic Energy Agency), Vienna (351 pp.).

Arnórsson, S., Gunnlaugsson, E., Svavarsson, H., 1983. The chemistry of geothermal waters in Iceland. III. Chemical geothermometry in geothermal investigations. Geochim. Cosmochim. Acta 47, 567-577.

Baas Becking, L.G.M., Kaplan, I.R., Moore, D., 1960. Limits of the natural environment in terms of $\mathrm{pH}$ and oxidation-reduction potential. J. Geol. 68, 243-284.

Barnes, I., Back, W., 1964. Geochemistry of iron-rich groundwater of southern Maryland. J. Geol. 72, 435-447.

Beato, D.A.C., Viana, H.S., Davis, E.G., 2000. Avaliação e diagnóstico hidrogeológico dos aqüíferos de águas minerais do Barreiro do Araxá, MG, Brasil. Proc. I Joint World Congress on Groundwater, Fortaleza, pp. 1-20.

Bertolo, R., Hirata, R., Fernandes, A., 2007. Hidrogeoquímica das águas minerais envasadas no Brasil. Rev. Bras. Geosci. 37, 2-15.

Birke, M., Rauch, U., Lorenz, H., Kringel, R., 2010a. Distribution of uranium in German bottled and tap water. J. Geochem. Explor. 107, 272-282.

Birke, M., Rauch, U., Harazim, B., Lorenz, H., Glatte, W., 2010b. Major and trace elements in German bottled water, their regional distribution, and accordance with national and international standards. J. Geochem. Explor. 107, 245-271.

Bityukova, L., Petersell, V., 2010. Chemical composition of bottled mineral waters in Estonia. J. Geochem. Explor. 107, 238-244.

Bonotto, D.M., 2006. Hydro(radio)chemical relationships in the giant Guarani aquifer, Brazil. J. Hydrol. 323, 353-386.

Bonotto, D.M., 2016. The dissolved uranium concentration and ${ }^{234} U /{ }^{238} U$ activity ratio in groundwaters from spas of southeastern Brazil. J. Environ. Radioact. http://dx.doi.org/ 10.1016/j.jenvrad.2016.03.009. 
Brenčič, M., Vreča, P., 2010. The use of a finite mixture distribution model in bottled water characterization and authentication with stable hydrogen, oxygen and carbon isotopes - case study from Slovenia. J. Geochem. Explor. 107, 391-399.

Brenčič, M., Ferjan, T., Gosar, M., 2010. Geochemical survey of Slovenian bottled waters. J. Geochem. Explor. 107, 400-409.

Brookins, D.G., 1988. Eh-pH Diagrams for Geochemistry. Springer-Verlag, Berlin (176 pp.).

Brownlow, A.H., 1996. Geochemistry. second ed. Prentice-Hall, Englewood Cliffs (580 pp.).

Cicchella, D., Albanese, S., De Vivo, B., Dinelli, E., Giaccio, L., Lima, A., Valera, P., 2010. Trace elements and ions in Italian bottled mineral waters: identification of anomalous values and human health related effects. J. Geochem. Explor. 107, 336-349.

CPRM (Brazilian Geological Survey), 1999. Projeto Circuito das Águas do Estado de Minas Gerais - Estudos Geoambientais das Fontes Hidrominerais de Águas de Contendas, Cambuquira, Caxambu, Lambari e São Lourenço. CPRM, Belo Horizonte (142 pp.).

CPRM (Brazilian Geological Survey), 2008. Geologia da Folha Varginha SF.23-V-D-VI. CPRM, Brasilia (99 pp.).

CPRM (Brazilian Geological Survey), 2012. A indústria brasileira de água mineral. http:// www.cprm.gov.br/.

Cruz, W.B., Peixoto, C.A.M., 1989. Águas termais de Poços de Caldas, MG: estudo experimental das interações água-rocha. Rev. Bras. Geosci. 19, 76-86.

Custodio, E.G., LLamas, M.R., 1976. Hidrologia Subterranea (T. 1). first ed. Omega, Barcelona.

Davenward, S., Bentham, P., Wright, J., Crome, P., Job, D., Polwart, A., Exley, C., 2013. Silicon-rich mineral water as a non-invasive test of the 'aluminum hypothesis' in Alzheimer's disease. J. Alzheimers Dis. 33 (2), 423-430.

del Rey, A.C., 1989. Estudo hidrogeotérmico da região de Águas de Lindóia, Amparo e Socorro-nordeste do Estado de São Paulo (Ms Dissertation) USP-University of São Paulo, São Paulo (124 pp.).

Demetriades, A., 2010. General ground water geochemistry of Hellas using bottled water samples. J. Geochem. Explor. 107, 283-298.

DFPM (Division for Supporting the Mineral Production), 1966. The Mining Code, the Mineral Waters Code and how Applying Research in a Mineral Deposit. eighth ed. DFPM, Rio de Janeiro.

Dinelli, E., Lima, A., De Vivo, B., Albanese, S., Cicchella, D., Valera, P., 2010. Hydrogeochemical analysis of Italian bottled mineral waters: effects of geology. J. Geochem. Explor. 107, 317-335.

Dotsika, E., Poutoukis, D., Raco, B., Psomiadis, D., 2010. Stable isotope composition of Hellenic bottled waters. J. Geochem. Explor. 107, 299-304.

Dušan, B., Jozef, K., Igor, S., Peter, M., Pavel, L., Daniel, P., Jarmila, B., Daniel, M., 2010. Mineral waters in Slovakia - evaluation of chemical composition stability using both historical records and the most recent data. J. Geochem. Explor. 107, 382-390.

Ebert, H., 1955. Sedimentos metamórficos de origem clástica e sua significação para o esclarecimento da estrutura geológica do Escudo Cristalino Brasileiro. Engenharia Mineração Metal. 22, 39-40.

Edmunds, W.M., Smedley, P.L., 2013. Fluoride in natural waters. In: Selinus, O., Alloway, B., Centeno, J.A., Finkelman, R.B., Fuge, R., Lindh, U., Smedley, P.L. (Eds.), Essentials of Medical Geology. Springer, Heidelberg, pp. 311-336.

Ellert, R., 1959. Contribuição à geologia do maciço alcalino de Poços de Caldas. Bol. Geol. FFCL USP 237, 5-64.

Eupedia, 2016. Mineral analysis of a few European mineral water brands. http://www. eupedia.com/europe/european_mineral_waters.shtml.

Faure, G., 1998. Principles and Applications of Geochemistry. second ed. Prentice-Hall, Upper Saddle River, NJ (600 pp.).

Fournier, R.O., 1977. Chemical geothermometers and mixing models for geothermal systems. Geothermics 5, 41-50.

Frengstad, B.S., Lax, K., Tarvainen, T., Jæger, Ø., Wigum, B.J., 2010. The chemistry of bottled mineral and spring waters from Norway, Sweden, Finland and Iceland. J. Geochem. Explor. 107, 350-361.

Fugedi, U., Kuti, L., Jordan, G., Kerek, B., 2010. Investigation of the hydrogeochemistry of some bottled mineral waters in Hungary. J. Geochem. Explor. 107, 305-316.

Garrels, R.M., 1959. Mineral Equilibria. Addison-Wesley Reading, Mass. (349 pp.).

Garrels, R.M., Christ, C.L., 1965. Minerals, Solutions and Equilibria. Harper \& Row, New York (453 pp.).

Gibson, S.A., Thompson, R.N., Dickin, A.P., Leonardos, O.H., 1995a. High-Ti and low-Ti mafic potassic magmas: key to plume-lithosphere interactions and continental flood basalt genesis. Earth Planet. Sci. Lett. 136, 149-165.

Gibson, S.A., Thompson, R.N., Leonardos, O.K., Dickin, A.P., Mitchell, J.G., 1995b. The Late Cretaceous impact of the Trindade mantle plume - evidence from large-volume, mafic, potassic magmatism in SE Brazil. J. Petrol. 36, 189-229.

Godoy, J.M., Amaral, E.C.S., Godoy, M.L.D.P., 2001. Natural radionuclides in Brazilian mineral water and consequent doses to the population. J. Environ. Radioact 53,175-182.

Gomes, C.B., Comin-Chiaramonti, P., 2005. Some notes on the Alto Paranaíba igneous province. In: Comin-Chiaramonti, P., Gomes, C.B. (Eds.), Mesozoic to Cenozoic Alkaline Magmatism in the Brazilian Platform. EDUSP, São Paulo, pp. 317-340.

Hach, 1992. Water Analysis Handbook. second ed. Hach Co., Loveland (831 pp.)

Hem, J.D., 1985. Study and interpretation of the chemical characteristics of natural waters. U.S. Geological Survey, Water-Supply Paper 2254, third ed..

Holmes, D.,.C., Pitty, A.E., Noy, D.J., 1992. Geomorphological and hydrogeological features of the Poços de Caldas caldera analogue study sites. J. Geochem. Explor. 45, 215-247.

Hounslow, A.W., 1995. Water Quality Data - Analysis and Interpretation. Lewis Publishers, Boca Raton (397 pp.).

Hydrogeologic, W., 2003. AquaChem User's Manual: Water Quality Data Analysis, Plotting \& Modeling. Waterloo Hydrogeologic, Waterloo (276 pp.).

IPT (Technological Research Institute of São Paulo State), 1981,. Geological Map From São Paulo State: Scale 1:500.000. Monographs. IPT, São Paulo (94pp.).
Kimmelmann, A.A., Yoshinaga, S., Murakami, H., Mattos, J.A., 1987. Novos aspectos hidrogeológicos, hidroquímicos e isotópicos das águas termominerais de Águas de São Pedro no Estado de São Paulo. Proc. VII Simp. Bras. de Hidrologia e Rec. Hídricos, Salvador, pp. 26-41.

Krauskopf, K.B., Bird, D.K., 1995. Introduction to Geochemistry. McGraw-Hill Inc., New York (647 pp.).

Lourenço, C., Ribeiro, L., Cruz, J., 2010. Classification of natural mineral and spring bottled waters of Portugal using principal component analysis. J. Geochem. Explor. 107, 362-372.

Mente, A., 2008. Mapa Hidrogeológico do Brasil. In: Feitosa, F.A.C., Manoel Filho, J., Feitosa, E.C., Demetrio, J.G. (Eds.), Hidrogeologia - conceitos e aplicações. CPRM-LABHID, Rio de Janeiro, pp. 31-48.

Milani, E.J., 2004. Comentários sobre a origem e evolução tectônica da Bacia do Paraná. In Mantesso-Neto, V., Bartorelli, A., Carneiro, C.D.R., Brito-Neves, B.B. (Eds.), Geologia do Continente Sul-Americano: Evolução da Obra de Fernando Flávio de Almeida. Beca, São Paulo, pp. 265-279.

Mourão, B.M., 1992. Medicina hidrológica - Moderna terapêutica das águas minerais e estâncias de cura. Secretaria Municipal de Educação, Poços de Caldas,

Oliveira, J., Mazzilli, B.P., Sampa, M.H.O., Silva, B., 1998. Seasonal variations of Ra-226 and Rn-222 in mineral spring waters of Águas da Prata-Brazil. Appl. Radiat. Isot. 49 (4) 423-427.

Oliveira, J., Mazzilli, B., Costa, P., Tanigawa, P.A., 2001. Natural radioactivity in Brazilian bottled mineral waters and consequent doses. J. Radioanal. Nucl. Chem. 249 (1), 173-176.

Peh, Z., Šorša, A., Halamić, J., 2010. Composition and variation of major and trace elements in Croatian bottled waters. J. Geochem. Explor. 107, 227-237.

Petrović, T., Zlokolica-Mandić, M., Veljković, N., Vidojević, D., 2010. Hydrogeological conditions for the forming and quality of mineral waters in Serbia. J. Geochem. Explor. 107, 373-381.

Piper, A.M., 1944. A graphic procedure in the geochemical interpretation of water analyses. Trans. Am. Geophys. Union 25, 914-928.

Reddy, D.V., Nagabhushanam, P., Sukhija, B.S., Reddy, A.G.S., Smedley, P., 2010. Fluoride dynamics in the granitic aquifer of the Wailapally watershed, Nalgonda District, India. Chem. Geol. 269, 278-289.

Renne, P.R., Ernesto, M., Pacca, I.G., Coe, R.S., Glen, J.M., Prévot, M., Perrin, M., 1992. The age of Paraná flood volcanism, rifting of Gondwanaland, and the Jurassic-Cretaceous boundary. Science 258, 975-979.

Ritter, S.M., 2012. Geothermometry on Natural Spring Waters From Poços de Caldas Minas Gerais, Brazil. Monograph. University of Heidelberg, Heidelberg (56 pp.)

Schoeller, H., 1962. Les eaux souterraines. Masson \& Cie, Paris (642 pp.).

Schorscher, J.H.D., Shea, M.E., 1992. The regional geology of the Poços de Caldas alkaline complex: mineralogy and geochemistry of selected nepheline syenites and phonolites. J. Geochem. Explor. 45, 25-51.

SEBRAE (Service for Supporting the Small Businesses in São Paulo State), 2012o. Comércio de água mineral. http://www.sebrae-sc.com.br/ideais/default.asp?vcdtexto= $31586 \& \% 5 \mathrm{E} \% 5 \mathrm{E}>$.

Serra, S.H., 2009. Águas minerais do Brasil. Millenium Editora, Campinas (272 pp.).

Smedley, P.L., Nicolli, H.B., Macdonald, D.M.J., Barros, A.J., Tullio, J.O., 2002 Hydrogeochemistry of arsenic and other inorganic constituents in groundwaters from La Pampa, Argentina. Appl. Geochem. 17, 259-284.

Smith, E.A., Mayfield, C.I., Wong, P.T.S., 1977. Physical and chemical characterization of selected natural apatites in synthetic and natural aqueous solutions. Water Air Soil Pollut. 8, 401-415.

Szikszay, M., 1981. Hidrogeoquímica das fontes de Águas da Prata, Estado de São Paulo (Post PhD Thesis) USP-University of São Paulo, São Paulo (193 pp.).

Thiede, D.S., Vasconcelos, P.M., 2010. Paraná flood basalts: rapid extrusion hypothesis confirmed by new ${ }^{40} \mathrm{Ar} /{ }^{39} \mathrm{Ar}$ results. Geology 38 (8), 747-750.

Thompson, R.N., Gibson, S.A., Mitchell, J.G., Dickin, A.P., Leonardos, O.K., Brod, J.A., Greenwood, J.C., 1998. Migrating Cretaceous-Eocene magmatism in the Serra do Mar alkaline province, SE Brazil: melts from the deflected Trindade mantle plume? J. Petrol. 39, 1493-1526.

Traversa, G., Gomes, C.B., Brotzu, P., Buraglini, N., Morbidelli, L., Principato, M.S., Ronca, S., Ruberti, E., 2001. Petrography and mineral chemistry of carbonatites and mica-rich rocks from the Araxá complex (Alto Paranaíba Province, Brazil). An. Acad. Bras. Cienc. 73, 71-98.

Turner, S., Regelous, M., Kelley, S., Hawkesworth, C.J., Mantovani, M.S.M., 1994 Magmatism and continental break-up in the South Atlantic: high precision ${ }^{40} \mathrm{Ar}-{ }^{39} \mathrm{Ar}$ geochronology. Earth Planet. Sci. Lett. 121, 333-348.

Ulbrich, H.H.G.J., Vlach, S.R.F., Demaiffe, D., Ulbrich, M.N.C., 2005. Structure and origin of the Poços de Caldas alkaline massif. In: Comin-Chiaramonti, P., Gomes, C.B. (Eds.), Mesozoic to Cenozoic Alkaline Magmatism in the Brazilian Platform. EDUSP, São Paulo, pp. 367-418.

Van der Aa, M., 2003. Classification of mineral water types and comparison with drinking water standards. Environ. Geol. 44, 554-563.

Verma, S.P., Pandarinath, K., Santoyo, E., 2008. SolGeo: a new computer program for solute geothermometers and its application to Mexican geothermal fields. Geothermics 37, 597-621.

WHO (World Health Organization), 2011. Guidelines for Drinking Water Quality. fourth ed. WHO Press, Geneva.

Zanardo, A., 1987. Análise petrográfica e microestrutural das rochas da Folha de Águas de Lindóia. USP-University of São Paulo, São Paulo, Ms Dissertation (270 pp.).

Zobell, C.E., 1946. Studies on redox potential of marine sediments. Bull. Am. Assoc. Pet. Geol. 30, 477-509. 